\title{
Australia's Role in Gravitational Wave Detection
}

\author{
John S. Jacob ${ }^{1}$, Pablo Barriga ${ }^{1}$, David G. Blair ${ }^{1}$, Aidan Brooks ${ }^{2}$, \\ Ron Burman ${ }^{1}$, Raymond Burston ${ }^{3}$, Lee Chan ${ }^{1}$, XiuTing Chan ${ }^{1}$, \\ Eu-Jeen Chin ${ }^{1}$, Jong Chow ${ }^{4}$, David Coward ${ }^{1}$, Benedict Cusack ${ }^{4}$, \\ Glen de Vine ${ }^{4}$, Jerome Degallaix ${ }^{1}$, Jean-Charles Dumas ${ }^{1}$, \\ Angus Faulkner ${ }^{1}$, Florin Garoi ${ }^{1}$, Slawomir Gras ${ }^{1}$, Malcolm Gray ${ }^{4}$, \\ Murray Hamilton ${ }^{2}$, Matthew Herne ${ }^{1}$, C. Hollitt ${ }^{2}$, David Hosken ${ }^{2}$, \\ Eric Howell ${ }^{1}$, Li Ju${ }^{1}$, T. Kelly ${ }^{2}$, Ben Lee ${ }^{1}$, Chuen Ying Lee ${ }^{5}$, \\ Kah Tho Lee ${ }^{1}$, Antony Lun ${ }^{3}$, David McClelland ${ }^{4}$, Kirk McKenzie ${ }^{4}$, \\ C. Mow-Lowry ${ }^{2}$, Damien Mudge ${ }^{2}$, Jesper Munch ${ }^{2}$, Darren Paget ${ }^{1}$, \\ Sascha Schediwy ${ }^{1}$, Susan Scott ${ }^{4}$, Antony Searle ${ }^{4}$, Ben Sheard ${ }^{4}$, \\ Bram Slagmolen $^{4}$, Peter Veitch ${ }^{2}$, John Winterflood ${ }^{1}$, \\ Andrew Woolley ${ }^{1}$, Zewu Yan ${ }^{1}$ and Chunnong Zhao ${ }^{5}$ \\ ${ }^{1}$ School of Physics, University of Western Australia, Perth \\ jsjacob@physics.uwa.edu.au \\ ${ }^{2}$ Department of Physics, University of Adelaide, Adelaide \\ ${ }^{3}$ Department of Mathematics, Monash University, Melbourne \\ ${ }^{4}$ Department of Physics, Faculty of Science, Australian National University, Canberra \\ ${ }^{5}$ School of Computer and Information Science, Edith Cowan University, Perth
}

Received 2003 May 12, accepted 2003 May 19

\begin{abstract}
An enormous effort is underway worldwide to attempt to detect gravitational waves. If successful, this will open a new frontier in astronomy. An essential portion of this effort is being carried out in Australia by the Australian Consortium for Interferometric Gravitational Astronomy (ACIGA), with research teams working at the Australia National University, University of Western Australia, and University of Adelaide involving scientists and students representing many more institutions and nations. ACIGA is developing ultrastable high-power continuous-wave lasers for the next generation interferometric gravity wave detectors; researching the problems associated with high optical power in resonant cavities; opening frontiers in advanced interferometry configurations, quantum optics, and signal extraction; and is the world's leader in high-performance vibration isolation and suspension design. ACIGA has also been active in theoretical research and modelling of potential astronomical gravitational wave sources, and in developing data analysis detection algorithms. ACIGA has opened a research facility north of Perth, Western Australia, which will be the culmination of these efforts. This paper briefly reviews ACIGA's research activities and the prospects for gravitational wave astronomy in the southern hemisphere.
\end{abstract}

Keywords: gravitational waves

\section{Introduction}

Gravitational waves are ripples in space-time which carry energy and angular momentum at the speed of light. Predicted by the general theory of relativity, there has been to date only indirect evidence for their existence, through the observation of energy loss from binary pulsars (Weisberg \& Taylor 1984). Numerous experiments have confirmed the underlying theory of general relativity to a high degree of precision. Yet the direct observation of gravitational waves is still necessary for the wave solutions of Einstein's field equations to be fully investigated. More importantly, however, the ability to directly detect gravitational waves will also create a new kind of astronomy.

Researchers in Australia are deeply involved in the pursuit of this discovery. Throughout the 1980s and 1990s, scientists at the University of Western Australia (UWA) were leaders in the field of resonant bar gravitational wave detectors. Work that will have a direct and vital impact on the world's second generation of gravitational wave interferometer detectors is now underway across Australia. Ultrastable, high-power continuous-wave lasers are being developed at the University of Adelaide (UA). Advanced interferometer design, signal extraction, data analysis, and quantum optics projects are underway at the Australian National University (ANU). UWA is now a world leader in high-performance vibration isolation and suspension design, as well as conducting research in the areas of thermal noise, thermal lensing effects, optical spring and radiation pressure effects, and astronomical sources of gravitational waves. These various projects come together as the Australian Consortium for Interferometric Gravitational Astronomy (ACIGA), which is collaborating with the US Laser Interferometer Gravitational Wave Observatory (LIGO) project to develop key technologies for the 
Advanced LIGO program. ACIGA has built a research facility $80 \mathrm{~km}$ north of Perth, named the Australian International Gravitational Observatory (AIGO).

\section{Gravity Waves: A New Spectrum for Astronomical Observation}

Since antiquity, our only source of information about the stars has been through their electromagnetic radiation. Developments in technology have allowed us to expand our window on the Universe from strictly visible radiation to infrared, microwave, radio, ultraviolet, X-ray, and gamma-ray radiation. Gravitational wave astronomy offers an entirely new spectrum of radiation through which to explore the Universe.

Just as Maxwell's equations predict oscillating electric and magnetic fields which, once initiated, self-propagate at the speed of light, Einstein's general theory of relativity predicts the propagation of waves of gravitational and gravitomagnetic fields at the speed of light. Similar to the way electromagnetic waves are created by the acceleration of charges, gravitational waves are created by the acceleration of mass. Since there is only one kind of gravitational charge (compared to two kinds of electric charges: positive and negative), and since conservation of momentum disallows unopposed acceleration, periodic mass dipoles are not possible. However, periodic mass quadrupole moments are possible in the form of a rotating non-spherically symmetric mass, such as a 'twirling' dumbbell. Gravitational wave luminosity is proportional to the square of the third time derivative of the mass quadrupole moment and to the constant $G / c^{5}$, where $G$ is the universal gravitational constant and $c$ is the speed of light. This is an extraordinarily small number.

The significance of gravitational waves to astronomy is impossible to predict. Because gravitational waves are created by bulk motions of matter, and because normal matter is almost totally transparent to gravitational wave radiation, they may allow us to listen in on regions and processes that are otherwise hidden from view, such as the inner regions of a supernova core collapse. Whereas electromagnetic telescopes are our eyes on the Universe, gravitational wave detectors constitute ears which will allow us to hear for the first time the 'sounds' produced by the Universe.

This paper's purpose is to review Australian research efforts in support of the international search for gravitational waves. The remainder of this introduction will present Australia's historical involvement in gravitational wave research, and will outline the current and future techniques being developed for gravitational wave astronomy. The following section will present more detailed information about likely astronomical gravitational wave sources, which are also the subject of much study in this country. The third section will discuss very long baseline laser interferometry in some detail, widely regarded as technically the best chance for gravitational wave detection within the next 10 years. Following this, an in-depth review of Australia's role in key scientific areas relating to gravitational wave research is presented. Finally, the AIGO will be introduced with its current state of development, its near-term role in international gravitational wave research and high-power laser interferometry development, and its long-term prospects as the premier southern hemisphere gravitational wave antenna.

\section{Predicting and Searching for Gravity Waves since 1915}

One simple principle underlies the design of all gravitational wave detectors. Gravitational waves are waves of gravity gradient, or in other words tidal forces, which create tidal deformations analogous to those exerted by the static tidal force of the Moon on the Earth. The signal is manifested as an oscillatory strain $h=\Delta L / L$, which can be detected through a simple linear strain measurement or through a differential measurement of strain between two orthogonal directions. The transduction of the gravitational wave strain may occur through the coupling of the signal into the acoustic phonon field within a large mass, or through the coupling into an electromagnetic photon field between freely suspended masses. Ideally, the size of the acoustic detector should be of order half an acoustic (phonon) wavelength of the expected signal frequency, while for an electromagnetic detector the scale should be half the electromagnetic (photon) wavelength of the expected signal frequency.

For gravitational waves in the audio frequency band originating from processes associated with stellar mass black holes and neutron stars, the above designs dictate the use of acoustic resonators of a few metres' scale, and of electromagnetic detectors of a few hundred kilometres' scale. For lower frequency signals associated with nonrelativistic binary stars and supermassive black holes the scale increases by about four orders of magnitude.

\section{Resonant Bar Detectors}

Both acoustic and electromagnetic detection were defined in the early years of gravity wave research, though acoustic detectors were the first to be developed (Weber 1960). A resonant bar detector consists of a large, vibration-isolated suspended mass for which the fundamental resonant frequency is the same as the gravitational wave frequency of interest. A gravitational wave of that frequency will excite vibrations in the bar which can be detected. Weber's bar was made of aluminium due to its high phonon velocity and low acoustic losses. Since then, many variations and improvements to resonant bar technology have been made.

In Perth, technology development for the first acoustic detector began in 1976. The 1.5 tonne cryogenic niobium bar detector, Niobe, was developed during the 1980s at UWA (Linthorne et al. 1990). In 1993 Niobe was shown to have the lowest 'noise temperature' of any detector to date (Blair et al. 1995). From 1993 to 1997 the antenna was operated almost continuously in search of gravitational waves, and in 1997 again set the record low noise temperature (Tobar et al. 1999). 
In 2000 Niobe was operated again with an improved amplifier, demonstrating yet another new record low noise temperature (Coward et al. 2002a). However, in 1999 Australian Research Council (ARC) funding for Niobe was discontinued, making long-term continuous operation impossible. Since then, all gravitational wave research at UWA has concentrated on technology development for advanced laser interferometer gravitational wave detectors.

\section{Interferometry as a More Promising Detection Technique}

The sensitivity of an acoustic detector is fundamentally limited by its size, which is in turn limited by the speed of sound within the bar material and the requirement that the fundamental resonant frequency of the bar match a desired detection frequency. These considerations have so far limited bars to just a few metres in length for detecting signals of frequency up to about $1 \mathrm{kHz}$. If the detection method were to use photons instead of phonons, the scale of the detector having the same upper frequency limit could be increased by a factor of $10^{5}$. Given that gravitational waves are strain waves, this would increase the detector displacement by the same factor. A detector of this scale $(150 \mathrm{~km})$ would be analogous to a half-wave electromagnetic dipole antenna. In practice, Earth-based detectors are limited to a few kilometres in length due to economics as well as the curvature of the earth. In any case, the advantages of measuring differential strain using light instead of sound are numerous.

A Michelson interferometer is capable of sensing differential changes in optical path lengths of much less than a wavelength of light over the entire length of each arm. When the two arms of the interferometer form a $90^{\circ}$ angle, the arm lengths are affected by a gravitational wave in antiphase due to gravitational waves' quadrupole polarisation, naturally doubling the single baseline sensitivity. An interferometer measures the relative strain of its two arms by comparing the optical path lengths between a beamsplitter and mirrors which terminate each arm, referred to as 'test masses'. The sensitivity of interferometry is sufficient to reduce the necessary size of detectors to only a few kilometres, making them much easier to construct on the surface of the Earth. Instruments such as these, though complex and largely still under development, offer the best opportunity for detecting gravitational waves in the 100 to $1000 \mathrm{~Hz}$ range.

Space-based interferometers would have fewer size restrictions. Without the seismic noise constraints of Earth-based detectors, they could provide detection of gravitational waves in the $10^{-1}$ to $10^{-5} \mathrm{~Hz}$ range. The Laser Interferometer Space Antenna (LISA) project, a joint proposal of NASA and ESA, intends to do just that.

There are four major Earth-based interferometric detection efforts underway outside Australia. The LIGO project in the US is a joint project of CalTech, MIT, and the National Science Foundation, and operates two $4 \mathrm{~km}$ baseline interferometers at sites in Hanford, Washington, and
Livingston, Louisiana. GEO is a joint German-British $600 \mathrm{~m}$ baseline interferometer near Hanover, Germany. VIRGO is a joint French-Italian project near Pisa, Italy, with a $3 \mathrm{~km}$ arm length. TAMA in Japan is a $300 \mathrm{~m}$ interferometer.

These first generation facilities serve two purposes. The first is to determine the upper limits on the strength of gravitational wave emissions, and, with luck, to detect rare, high strength signals. The second is to push the development of the necessary technologies for achieving a sensitivity that will guarantee routine gravitational wave detection. Detection of gravitational waves with this first group of instruments is likely only in the event of a Galactic supernova or a relatively nearby neutron star/neutron star in-spiral event occurring while two or more detectors are operational in coincidence. While it is likely that there will be confirmed gravitational wave detection in the near future, technology must improve before gravitational wave detection will become a regular part of astronomical inquiry.

To that end, LIGO has already made plans for the next generation interferometers, called Advanced LIGO. An essential part of the Advanced LIGO program is the ability to operate with high optical power in the interferometer. This requires extremely 'clean' high-power lasers that can produce about 100 watts of optical power at a single wavelength and in a single spatial mode $\left(\mathrm{TEM}_{00}\right)$. Since the optical power is built up in resonance to levels on the order of megawatts, advanced optics are required that can cope with such high incident optical power. Very low absorption substrates and coatings and very high reflectivity mirrors are needed, as well as a way of handling the 'thermal lensing' effect - the change in focal length caused by local heating through absorption of light in the optics.

ACIGA plays two vital roles in gravitational wave research. The first is to collaborate with LIGO in the development of high optical power technologies. The AIGO site at Gingin, $80 \mathrm{~km}$ north of Perth, Western Australia, will initially serve as a research and testing facility for high-power optics and thermal lensing, with several experiments now planned on an $80 \mathrm{~m}$ baseline suspended optical cavity. Its second role is to develop the AIGO site into the first southern hemisphere gravitational wave observatory, completing the global network of gravitational wave detectors. Such a network is essential for coincidence checking of gravitational wave signals and for determining the likely origins of gravitational wave signals.

\section{Astronomical Sources of Gravitational Waves}

This section provides a brief overview of several potentially observable types of gravitational wave sources based on current and planned interferometric detectors. It is not intended to be an exhaustive list but rather to highlight the rich diversity of possible sources. A more comprehensive and rigorous treatment of gravitational wave sources can be found in Cutler \& Thorne's (2002) review. 
Astrophysical sources of gravitational waves can be broadly categorised as being either continuous or burst sources. Continuous sources are ones yielding slowly evolving emission, with a characteristic evolution time that is very long compared with the observation time. By choosing one year as a practical observation time, sources that could be considered continuous include compact binary systems in the gradual in-spiral phase (due to gravitational wave radiation reaction) prior to the last few minutes of their evolution. The final seconds before coalescence will produce the strongest gravitational waves in a type of burst signal often termed a chirp because of the rapidly increasing amplitude and frequency as the compact objects coalesce. The most intensely studied theoretical sources of this type involve black holes and neutron stars.

Wide-band detectors can potentially detect the gravitational wave chirp signals from neutron star/neutron star coalescing binaries out to $300 \mathrm{Mpc}$, neutron star/black hole binaries $\left(\mathrm{M}_{\mathrm{bh}}<10 \mathrm{M}_{\odot}\right)$ out to $650 \mathrm{Mpc}$, and black hole/black hole binaries out to redshift $z=0.4$ (Cutler \& Thorne 2002). Rates for these events are calculated by extrapolating the local density of massive stars, based on observational data from our Galaxy, out to cosmological distances (Kalogera et al. 2001; Belczynski \& Kalogera 2001). Radio astronomy provides observational constraints on the number of neutron star/neutron star binaries using the observed numbers of binary pulsar systems, and recent estimates of event rates for gravitational wave detectors range from 1 to $800 \mathrm{yr}^{-1}$. For the case of neutron star/black hole mergers, there are no observations of pulsar-black hole systems, so detectability rates rely on population synthesis techniques to simulate the evolution of a population of progenitor binary systems. Kalogera et al. (2001) find neutron star/black hole event rates of $1-1500 \mathrm{yr}^{-1}$. For black hole/black hole mergers, similar methods yield rates of $30-4000 \mathrm{yr}^{-1}$, but Cutler \& Thorne (2002) point out that such calculations ignore the role of globular clusters in the formation of black hole/black hole binaries and that the lower limit may consequently be an order of magnitude higher.

Rapidly spinning pulsar neutron stars are potential continuous gravitational wave sources. When a neutron star reaches maximum stability in its final state, it possesses a crystallised solid crust. Because of spin the crust will have assumed an oblate axisymmetric shape due to centrifugal forces, with an axis that follows the spin axis. If its angular momentum deviates from this preferred axis, the neutron star will 'wobble' as it spins. Such a neutron star will emit gravitational waves at twice its rotational frequency. Pulsars with spin frequencies above $100 \mathrm{~Hz}$ are possible detectable sources of gravitational waves.

Sources with characteristic evolution times that are very short compared with the observation time can be considered as burst sources. A typical burst source example is a core-collapse supernova, for which the evolution time could be of order milliseconds. Because the core-collapse process involves relativistic compact mass motion, gravitational wave emission should be an intrinsic feature of these events. But modelling stellar core collapse is an extremely difficult and complex task. Early attempts to predict gravitational wave emission were semiquantitative, predicting a broadband emission lasting for a time of order milliseconds; see Eardley (1983) for an early review. Because of recent advances in supercomputing and computational techniques, we are just beginning to gain insight into the details of how gravitational wave emission might occur.

Stellar collapse certainly exhibits all of the necessary conditions for strong gravitational wave generation: large mass flow in a compact region (hundreds to thousands of kilometres) at relativistic speeds. However, these conditions are not sufficient to guarantee strong emission. In particular, the degree of asymmetry in collapse is not well understood. If stellar cores are rapidly rotating, instabilities can develop that are certain to drive strong gravitational wave emission.

The newborn neutron star may be dynamically unstable, resulting in a bar-mode instability. Recent simulations suggest that a fast spinning newborn neutron star may develop into a bar-shaped object with a radius that is large compared to the final neutron star radius (Shibata et al. 2000). A bar-mode instability is one of the more promising mechanisms by which a significant fraction of the collapsing system's energy can be emitted in gravitational waves. Bar-mode instabilities occur in objects whose rotational kinetic energy exceeds some fraction of their potential energy. Simulations show that general relativity enhances the instability to bar formation (Saijo et al. 2001) and that the bar will be long-lived. Gravitational waves from this source will contain information on the evolution of the bar mode and reveal the dynamics of the process through the frequency evolution of the gravitational waves. Only rough estimates exist for the detectability of these sources, but it is plausible that they could be detected out to the Virgo cluster (15-20 Mpc) at a rate of several per year.

Unfortunately, supernova rates are too low in our Galaxy for us to expect to observe a Galactic supernova during a period of even tens of years' observation time (e.g. Dragicevich et al. 1999). But if some or all of the processes discussed above are common to a large fraction of core-collapse events, then we could possibly observe several events per year, assuming sensitivities of the planned second generation detectors.

Gamma-ray bursts have been observed for decades but their true nature is just beginning to come to light. They are associated with host galaxies that are not particularly luminous; the galaxies appear blue (Hogg \& Fruchter 1999) and the location of the optical transient is never very distant from the galaxy centre, in agreement with the idea that long gamma-ray bursts (those lasting $>2 \mathrm{~s}$ ) are associated with massive and short-lived progenitors (for a recent review see Djorgovski et al. 2001). The central engine could be a fast-spinning black hole surrounded by a very dense (neutron-dense) torus. In this case the available energy sources, apart from gravitational radiation, are in the form of neutrinos, accretion of the torus 
material onto the black hole, and rotation of both the torus and the hole. Van Putten (2001) has developed a model in which the accretion disk of the magnetised newborn black hole becomes highly compressed, resulting in an accreting torus. The torus might develop large nonaxisymmetries, or 'lumpiness', in the process emitting gravitational waves. He has shown that about $10 \%$ of the black hole's spin energy could be radiated away in gravitational waves. Estimates for the amplitude of this source yield a gravitational wave energy emission equivalent to $0.3 \mathrm{M}_{\odot}$, radiating in a frequency range around $1 \mathrm{kHz}$. Coincidence analysis between electromagnetic emissions and gravitational wave signals could provide a powerful probe for investigating these enigmatic phenomena further.

Several current theories of early Universe cosmology, including inflationary and grand unified theories, predict strong gravitational wave emission from the epoch of graviton decoupling. Detection of a gravitational wave background from the early Universe would have a profound impact on early Universe cosmology and on highenergy physics, opening up a new window to explore very early times in the evolution of the Universe, and correspondingly high energies that will never be accessible by other means. Relic gravitational waves carry unique information from the primordial plasma, providing a 'snapshot' of the state of the Universe at that epoch: cosmological gravitational waves could probe deep into the very early Universe. The gravitational wave background from the primordial Universe is a stochastic background, random in amplitude with an energy density spread across a large bandwidth.

There is another type of gravitational wave stochastic background - one comprised of the cumulative emissions from astrophysical sources throughout the Universe (e.g. Ferrari et al. 1999a, 1999b). This background is very interesting, because it may be continuous across certain frequency bands but comprised of discrete events within other bands. The background from massive binary systems may form the continuous component, with burst sources such as supernovae and gamma-ray bursts producing a gravitational wave background that can be described as 'popcorn noise'. Most of the sources, as observed in our frame, will be at cosmological distances with highly redshifted emissions. Coward et al. (2002b), part of the Australian consortium, have produced graphical and audio Monte Carlo simulations using some computed supernovae-generated gravitational wave waveforms as a basis.

\section{Principles of Laser-Interferometric Gravitational Wave Detection}

Numerous types of interferometers (e.g. Michelson, Fabry-Perot, and Sagnac), and many variations on these (e.g. power-recycling, signal recycling, dual-recycling, resonant recycling, open-area Sagnac, zero-area Sagnac, delay line, resonant sideband extraction, all-reflective) have been proposed and investigated as potential gravitational wave detectors. As can be expected, each variation offers an advantage in the form of suppressed sensitivity to certain sources of noise, and each comes with disadvantages that ultimately mean greater sensitivity to other sources of noise.

The configuration selection process becomes an exercise in determining which type of noise source can be adequately dealt with externally, and to which noise sources the interferometer must have the greatest immunity. When all noise sources, such as seismic noise, laser noise, suspension and optics thermal noise, and partial-vacuum scattering have been dealt with, most major interferometer configurations come out about equal in signal sensitivity and are limited by the same pair of complementary noise sources: photon shot noise and radiation pressure noise. In this realm, various tricks can be employed which pit one against the other, but to reduce both at the same time runs up against the standard quantum limit (SQL), the theoretical sensitivity limit imposed by the Heisenberg uncertainty principle.

\section{Reduction of Noise Sources}

Photon shot noise increases with signal frequency and can be reduced by using more photons (higher optical power). Radiation pressure noise is highest in the lower frequency range and increases with optical power. Thus, there is an optimal amount of optical power that depends on the required detection frequency. Also, the narrower the detection band, the better the optimisation can be made, until the SQL is reached.

Most current interferometer designs aim for a reasonably broad detection band of $100 \mathrm{~Hz}$ to $1 \mathrm{kHz}$, since this increases the likelihood of observing a gravitational wave event. At present, sensitivity is almost universally shotnoise limited; hence the push for higher optical power lasers and optics in future detectors. Sensitivities close to the SQL are thought to be sufficient to ensure routine gravitational wave detection. This assumes that other noise sources have been adequately dealt with.

Seismic Noise: By far the most predominant noise source affecting any Earth-based gravitational wave detector is seismic noise. This is the continuous, broadspectrum, multiple degree-of-freedom motion of the surface of the Earth caused by natural processes such as ocean waves, wind, animal movements, tidal forces, diurnal local heating, or tectonic activity, as well as by human activity such as road traffic, plant machinery, or foot traffic. Typical seismic amplitudes are given by $x_{\mathrm{s}} \approx \alpha f^{-2} \mathrm{mHz} z^{-1 / 2}$, where $\alpha \approx 10^{-6}$ to $10^{-9}$. This is at least eight to ten orders of magnitude larger than the expected test mass motions due to gravitational waves.

Interferometers are affected in two ways by seismic noise. Firstly, seismic noise can overwhelm by at least one million times any gravitational wave signals by disturbing the motions of the test masses at the frequencies of interest. Secondly, seismic vibrations can prevent an interferometer from locking onto a single interference fringe 
and can prevent optical cavities from attaining resonance, thus barring the interferometer from operating at all.

Reducing seismic noise in the detection band is a straightforward process of suspending test masses from successive stages of mechanical vibration attenuators in series. This must be done for each degree of freedom. For horizontal motion, the simplest arrangement is a series of pendulums and masses. For vertical motion, spring-mass combinations are used. Each stage is designed to have a resonant frequency well below the detection band. One drawback to this approach is the likelihood that the numerous internal modes in the pendulum suspension wires and vertical isolation springs could resonate at specific frequencies within the detection band.

There are two approaches to solving that problem. One is to identify and digitally remove these resonant peaks from the data during the data processing stage. The other, favoured by ACIGA, is to attend to the mechanical design of all isolator components to minimise the number of internal modes falling within the detection band. This has only become possible through a number of pioneering advances, including most significantly a vertical isolation spring with internal frequencies on order 1000 times higher than its fundamental spring-mass frequency (Winterflood et al. 2002). More will be said of this in Section 5.

Ensuring that the overall residual motions of the test mass are low enough to operate the interferometer is a more difficult problem. Each isolation stage has its own resonance which actually amplifies seismic noise at that frequency. Additionally, low-frequency seismic motions are not attenuated at all by the multiple-stage isolators mentioned above. Two revolutionary approaches to this are used by ACIGA. One is to construct pendulum isolation stages which can internally dissipate energy from their own periodic motion. Called 'self-damping' pendulums, this technique significantly reduces the amplitude of pendulum resonant modes, thereby reducing the residual test mass motion.

The other approach, used in conjunction with selfdamping, is to suspend the isolators from a very lowfrequency pre-isolator. The resonance of the pre-isolator (typically 0.025 to $0.1 \mathrm{~Hz}$ ) is easily controlled through active damping, effectively eliminating residual motion of the test mass at low frequencies. The pre-isolator also attenuates seismic motion that would otherwise excite both fundamental and higher order resonances in subsequent isolation stages.

Figure 1 shows the effect of adding low-frequency preisolation to one axis of motion. Five ordinary isolation stages alone (dotted line with five peaks) are adequate for removing seismic noise from the detection band (i.e. above $100 \mathrm{~Hz}$ ), but the isolator resonant peaks without pre-isolation result in unacceptably large residual motions of the test mass. The solid line shows the pre-isolation approach with one very low-frequency peak and four ordinary isolation stages. With pre-isolation, the isolator peaks are reduced to levels which the interferometer feedback

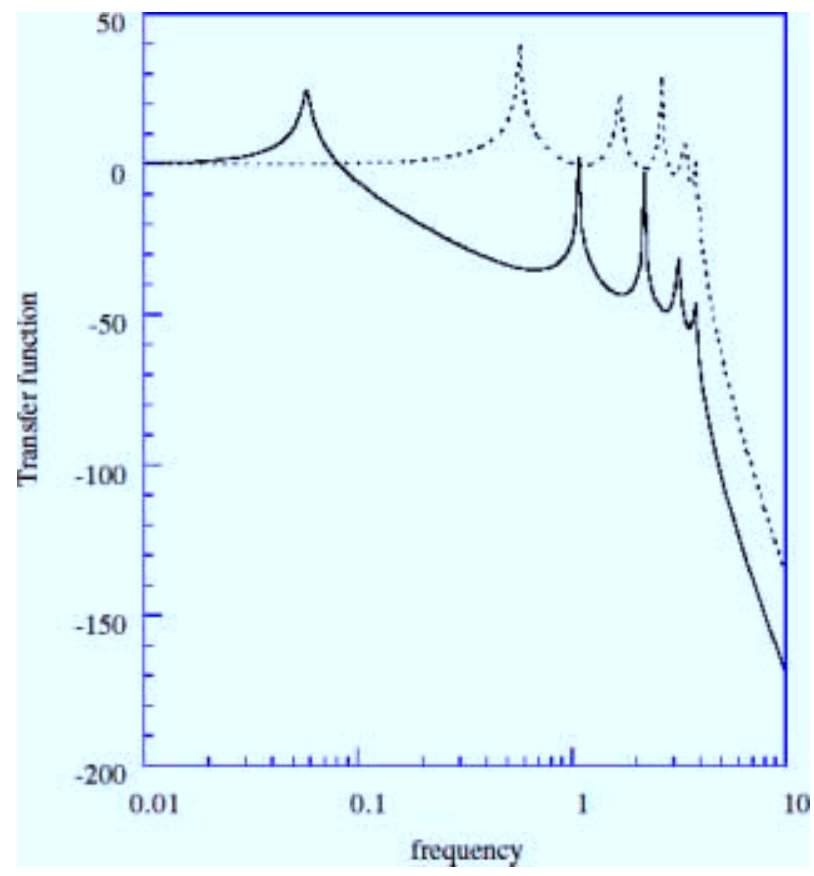

Figure 1 Effect on relative amplitudes of adding a very low frequency pre-isolation stage. Vertical units are ratio of output to input, in $\mathrm{dB}$. Two five degree-of-freedom systems are numerically modelled here to illustrate the effect of drastically reducing (by about one power of 10) the resonant frequency of one of the degrees of freedom, representing a pre-isolation stage.

controls can overcome, allowing the device to be locked onto a single interference fringe.

Also, care is taken to avoid any kind of moving, sliding, or pivoting joint or bearing in the mechanical design. Only elastic flexures are used. The reason for this is that the discontinuity between static and sliding friction in moving hinges creates impulses that excite resonances throughout the isolation and suspension structure. The broad-spectrum nature of such impulses means that resonances of any frequency are excited, including those which contaminate the detection band.

Laser Noise: Frequency fluctuations, intensity noise, and multiple TEM modes all emulate the signal produced by a gravitational wave in the interferometer output. Therefore, each of these must be dealt with in the design and selection of the input laser. High-power lasers are not all that rare today; however, producing continuous wave, highly monochromatic, low noise, single-mode $\left(\mathrm{TEM}_{00}\right)$ laser light of significant power still requires considerable effort.

Frequency stabilisation techniques are well developed for low-power lasers, and must now be adapted for use with higher power. Further immunity to frequency and phase noise in the interferometer is obtained through modulation and sideband extraction techniques. Phase noise due to multiple spatial modes is largely eliminated through the use of input and output mode cleaners - optical cavities in which only the particular desired mode is resonant. ACIGA is playing a key role in developing and demonstrating these laser techniques at high optical power. 
Thermal Noise: This noise source is divided into two separate phenomena: thermal noise of the test mass and thermal noise of the suspension. In either case, Brownian motion of the molecules comprising part of the interferometer can directly couple into the interferometer phase measurement and reduce the signal to noise $(S / N)$ ratio. The most straightforward approach to thermal noise is operating the entire interferometer at cryogenic temperatures (something proposed for detectors on the 10- to 20 -year horizon). However, this is not currently feasible due to the expense and complexity. A more basic approach therefore is taken, which in the long run will also enhance the effectiveness of the cryogenic approach.

Thermal noise has been studied within materials that have very high internal Q-factors, meaning materials that have very low 'internal friction' or 'material losses'; in other words, minimal 'phonon scattering' behaviour. It has been found that the thermal energy in such materials can be essentially sequestered within the internal resonance modes. The result is that with the exception of a few very narrow frequency bands which can easily be isolated at either the detection or data processing stages, the material is vibrationally very quiet. Materials with high Q-values are therefore highly desirable as test mass mirror substrates.

Fused silica and grown sapphire are two such substrate materials that are being studied for use in gravitational wave interferometers. The purity of the materials, the way they are grown, annealed, and polished, and the selection of coating materials all have an influence on the Q-factor and overall level of thermal noise, and are therefore subjects of intense study.

Suspension thermal noise is physically identical to test mass thermal noise, but is treated in a slightly different way. Rather than material selection being the essential solution, more attention is paid to the mechanical design of the suspension and its components. Again, the preferred approach is to sequester thermal energy into narrow, high$\mathrm{Q}$ resonances. Even more desirable are high-Q resonances which have frequencies that lie outside the detection band. More will be said of this in Section 4.

Vacuum System: Partial scattering of the laser light between test masses creates phase disturbances indistinguishable from the gravitational wave signal. It is therefore essential to operate the interferometer in as complete a vacuum as possible. LIGO attempts to operate in a vacuum of about $10^{-9}$ Torr. The difficulty and expense of achieving this in eight kilometres of one metre diameter pipe can be easily appreciated. Fortunately, AIGO's objectives can be accomplished at vacuum levels of just $10^{-7}$ Torr.

Cleanliness, especially with regard to hydrocarbon contaminants, is also an important issue within the vacuum envelope. Hydrocarbon molecules are easily forced into the surfaces of optics by radiation pressure. This causes reflectivity and other properties of the optics to degrade rapidly, and can cause extreme local heating of a substrate, possibly resulting in failure of the material. For these reasons, care is taken to eliminate all sources of

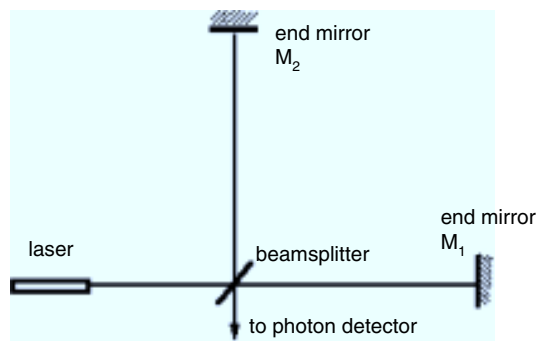

Figure 2 The basic Michelson configuration. The output of a laser is split into two arms which recombine at a photodetector in either constructive or destructive interference. Differences in the lengths of the arms of less than one wavelength of light are detectable as variations in intensity at the photodetector.

hydrocarbons in the vacuum vessels and pumps, as well as on the suspension and isolation hardware, and even within the laboratory building.

\section{Signal Extraction Principles in Interferometry}

A simple Michelson interferometer detector is shown schematically in Figure 2. The interferometer consists of three 'free masses' - one beamsplitter, and two test masses at right angles to form the end mirrors. These masses are vibration isolated and suspended so that at frequencies well above isolator resonances they can move freely as inertial test masses. When a gravitational wave passes it creates relative phase shifts between the two beams. These are read out as intensity variations in the interferometer output, giving information about the incoming gravitational wave.

For simplicity, consider the case of an incident gravitational wave perpendicular to the plane of the interferometer with a polarisation direction parallel to the interferometer arms. The passing wave will make one arm of the interferometer optically shorter and the other longer in half of the wave period, and reverse the contractionelongation process in the other half period. The relative change of optical lengths of the two arms $\Delta L=L 2-L 1$ can be described as a phase shift,

$$
\varphi=2 \pi / \lambda
$$

This results in a change in the interference pattern at the output of the beamsplitter. The relative difference in optical path $L$ is proportional to the arm length $\Delta L=h \times L$. Generally, an interferometer is sensitive to a linear combination of the two polarisation fields, and gravitation wave strain, $h$, in the above equation is

$$
h=F_{+} h_{+}+F_{\times} h_{\times}
$$

where $F_{+}$and $F_{\times}$are coefficients depending on the direction to the source and the orientation of the interferometer. Because the gravitational signal is extremely small, it is very difficult to monitor the small time-varying changes in the interference pattern due to the passing gravitational wave. In practice, the phase difference arising from the optical arm length variations is obtained by the so-called 
'nulling method'. The idea is to always keep the light returning from the two arms $180^{\circ}$ out of phase so that its output is always a dark 'fringe'. This is done by feeding the error signal back to the end mirrors via positional actuators to create a compensating phase shift such that the interferometer output remains 'locked' on a dark fringe. The control signals applied to the end mirrors contain the information of the gravitational wave disturbance. In this way the effect of power fluctuations in the laser beam can be minimised, the circulating optical power in the two arms is maximised, and the shot noise level is minimised.

Various modulation-demodulation techniques have been proposed and extensively studied for extracting the signal and locking the interferometer at a dark fringe. Schemes known as external modulation (Drever 1983; Man et al. 1990; Strain \& Meers 1991; Gray et al. 1996) and frontal modulation (Takahashi et al. 1994; Regehr et al. 1995; Flaminio \& Heitmann 1996; Ando et al. 1997) have been particularly investigated because their modulators are outside the interferometer. Thus, as opposed to internal modulation, these schemes avoid introducing losses or wavefront distortion within the interferometer (Billing et al. 1979). Although quantum noise-limited sensitivity has been achieved with internal modulation at low laser power level (Shoemaker et al. 1991; Stevenson et al. 1993, 1995), no large-scale laser interferometer detectors will use this configuration because it introduces losses and wavefront distortion. Instead, frontal modulation has been chosen and implemented in LIGO (Landry 2002), VIRGO (Bondu et al. 2000), GEO (Freise et al. 2002), and TAMA (Ando et al. 2001) projects due to its simplicity and robustness.

\section{Analysis of Interferometer Data}

Every gravitational wave detector possesses a threshold below which a decision on whether or not a gravitational wave 'event' occurred cannot be made. This threshold is determined by the detector noise and a probabilistic argument that either rejects or accepts that a gravitational wave signal is present in the data. A $S / N$ ratio is used to determine if a signal is present in a noise background, and can be optimised if prior knowledge of the signal is available. Matched filtering is a technique that relies on knowing the general form of the signal a priori, and optimises the $S / N$ by applying a replica (template) of the signal to a data set where the signal might be present. This template optimally enhances the $S / N$ if it matches the signal in both phase and amplitude.

Matched filter templates for likely sources are being developed based on current theoretical models for gravitational wave emission. Close compact binaries are one source type where the signal has been modelled in a post-Newtonian formalism that has allowed templates to be constructed. But the computational cost of matched filtering becomes enormous for a blind search if one has no knowledge of the source direction, masses of the binary objects, and initial phase and orientation angles of the binary orbit. This is a major computational problem that is being tackled by many groups in an effort to reduce the computational cost of close binary searches. One methodology uses a network of detectors to improve the detection confidence, but this is even more computationally expensive than a single detector search. The number of templates required is greatly reduced if one is searching for known sources, such as Galactic pulsars.

Observations of cosmic neutrino and astrophysical electromagnetic events may provide a means of performing coincidence tests between conventional astronomical observations and gravitational wave detectors.

For the case of random or stochastic backgrounds, such as the proposed primordial cosmological background, the method of cross-correlation is the optimal signal processing strategy. Each detector is assumed to be independent in detector noise, so that by combining the signal from several detectors the signal grows linearly while the noise grows as the square root of observation time, thus improving the $S / N$. This is because the noise is uncorrelated but the signal will be correlated if the detectors are separated by less than one reduced wavelength and the detectors are optimally aligned. This method has been implemented using the two LIGO detectors in 2002 and has provided the first upper limit on a cosmological background of gravitational waves. Cross-correlation may also be implemented to detect the presence of a gravitational wave background from astrophysical sources throughout the Universe (see Section 2).

\section{ACIGA Research Activities}

ACIGA has undertaken research in the four main interferometer 'subsystems': advanced configurations; lasers and optics; isolation, suspension, and thermal noise; and data analysis. Here we will briefly summarise our major achievements in these areas.

\section{Advanced Configurations}

Our research has focused on developing techniques to enhance the signal response using methods which are generally referred to as signal recycling, and to reduce noise by employing quantum optical techniques.

Signal Recycling: Figure 3(a) shows the proposed optical layout of a second generation laser interferometer gravitational wave detector. It differs from first generation interferometers (such as LIGO and VIRGO) by the presence of the signal recycling mirror. A passing gravity wave induces phase modulation sidebands on the carrier light circulating inside the arm cavities. At the main beamsplitter the carrier light is directed toward the power recycling mirror, where it is resonantly reflected back into the interferometer, to build up the stored power. This is termed power recycling (Drever 1983). On the other hand the signal sidebands are ejected at the main beamsplitter toward the signal mirror, where they are resonantly reflected back into the interferometer to enhance the signal. This effect is known as either signal recycling (SR) (Meers 1988) or resonant sideband extraction (RSE) (Mizuno et al. 1993) 
(a)

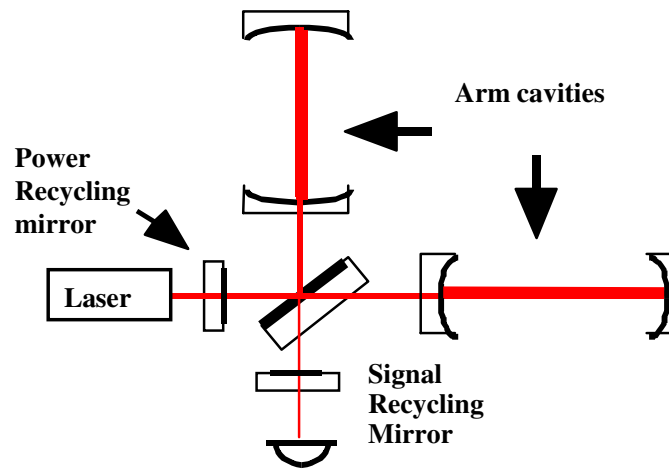

Signal Recycling/Resonant Sideband Extraction in a Michelson (b)

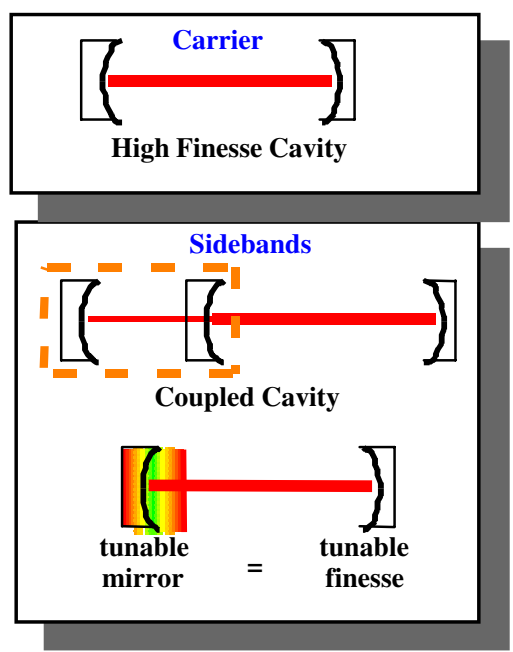

Figure 3 (a) Optical layout of a second generation laser interferometer showing power and signal recycling. (b) Principle of signal recycling: the carrier light builds up in the arm cavities and is not affected by the signal mirror. Build up of signal sidebands depends on resonant condition in the signal cavity.

depending on the resonant condition of the signal sidebands in the cavity formed by the signal mirror and the reflecting element in the interferometer arms; see Figure 3(b). From here on we will refer to the general class as signal recycling. The influence on the signal occurs only over a frequency range determined by the effective bandwidth of the signal recycling cavity, hence signal recycling modifies the frequency response of the interferometer.

One of the major problems to be solved to be able to implement signal recycling schemes is how to obtain signals to control the various optical cavity and Michelson degrees of freedom to maintain resonance and to extract the gravity wave signal. After examining various modulation schemes for interferometer control (Stevenson et al. 1993; Gray et al. 1996), we proposed a control scheme for a simple SR interferometer and experimentally verified the predicted frequency response. Following this, we demonstrated resonant sideband extraction on a Sagnac interferometer (Gray 1998). This work revealed the important result that the Sagnac arrangement is highly sensitive to beamsplitter imbalance when arm cavities are present.

More recently, we invented and demonstrated a control signal for RSE on a Michelson interferometer (Shaddock et al. 1998), the interferometer arrangement favoured for Advanced LIGO, which allows dynamic tuning of the peak response frequency. Our scheme, which uses a phase modulated carrier plus a subcarrier, now forms the basis of the control scheme which is likely to be adopted by LIGO.

The success of our RSE work leads to interferometers with tuneable peak response frequency. However, tuning the bandwidth requires the SR mirror to have a variable reflectivity. Changing the resonance condition of either a cavity or a Michelson interferometer varies the amplitude of the reflected field in both cases. This suggests either optical element could be used as a variable reflectivity mirror. Our analysis shows that the Michelson has the advantage that only the magnitude of the reflected field is changed, not its phase (in contrast to a cavity). This has advantages when designing an orthogonal control scheme (de Vine et al. 2002a) leading us to demonstrate the first tuneable bandwidth signal recycled interferometer (de Vine et al. 2002b). Work on the development of a suspended variable reflectivity mirror is in progress.

Quantum Optics: In 1980, Caves realised that the sensitivity of an interferometer could be improved by using squeezed states. Classical light is best represented by a coherent state: a state which the noise is the same in all quadratures and is set by the Heisenberg uncertainty principle. Squeezed states of light are states in which the noise in one quadrature has been reduced below the Heisenberg uncertainty principle level at the expense of an increase in the noise in the conjugate quadrature.

Squeezed states are made using non linear optical processes such as second harmonic generation and optical parametric oscillation. The world record for the generation of a squeezed continuous wave vacuum state is held by Lam et al. (1999) in which they measured $7 \mathrm{~dB}$ squeezing (inferring that up to $10 \mathrm{~dB}$ was generated).

If shot noise is the factor limiting the sensitivity, squeezing the phase quadrature of the vacuum input to the interferometer as demonstrated in Figure 4 would improve the sensitivity. This allows the SQL to be reached, at a lower optimum power level, but not surpassed. On the other hand, if the interferometer is limited by radiation pressure noise, light squeezed in the amplitude quadrature must be used to improve the sensitivity (again at best to the SQL).

We reported improvement in $S / N$ ratio of a power recycled interferometer (as depicted in Figure 4(a)) by injecting a squeezed vacuum state (McKenzie et al. 2002). Results are summarised in Figure 5. Using a benchtop scale Michelson interferometer, with a shot noise limited signal generated by modulating the piezo at $5.46 \mathrm{MHz}$, we injected a $3 \mathrm{~dB}$ squeezed state via the empty beamsplitter 


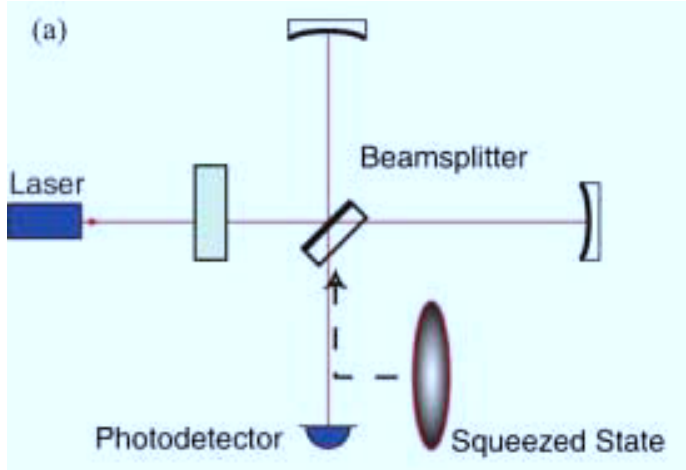

(b)

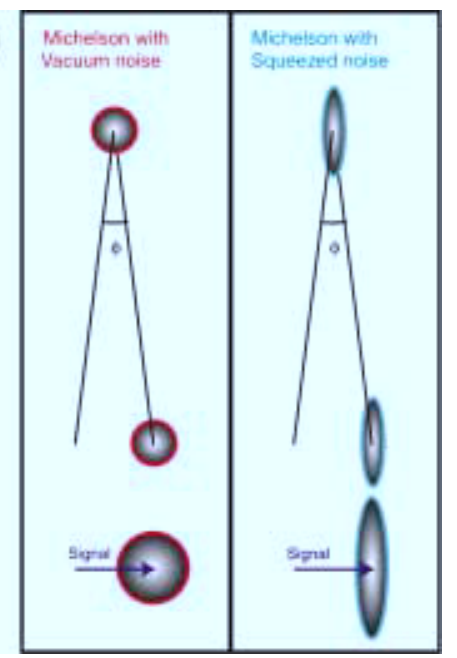

Figure 4 (a) A power recycled Michelson interferometer showing where a squeezed vacuum state is injected to modify quantum noise. (b) The lines represent the magnitude of the electric field phasors emerging from each of the interferometer arms which interfer at the beamsplitter. The phase difference represents the signal. On the left the vacuum state is a coherent state. On the right, a squeezed vacuum state is injected, showing the improvement in $S / N$ achieved by using squeezing (or alternatively that a smaller signal could be measured).

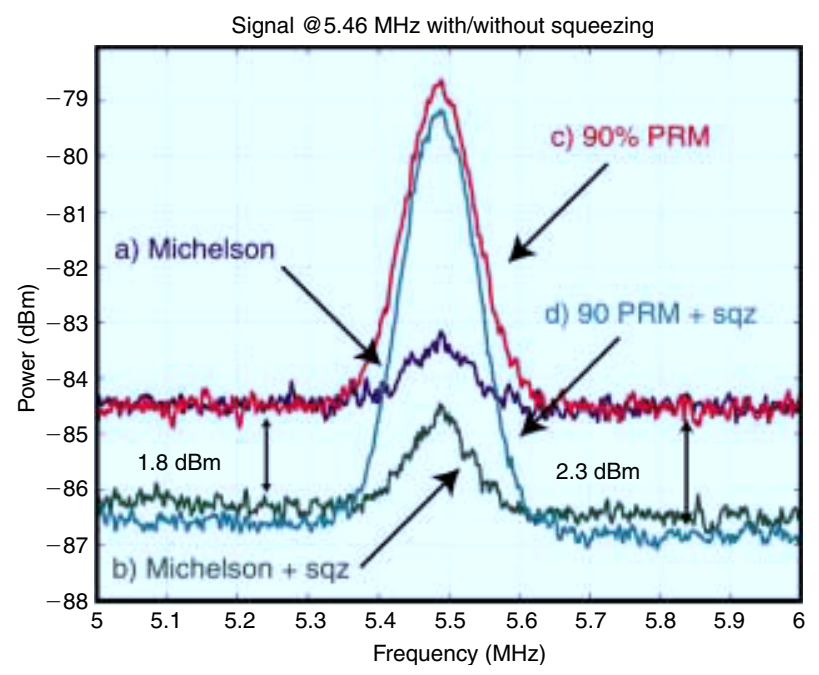

Figure 5 Signal to noise curves from McKenzie et al. (2002). There are four traces: smallest peak is for the basic interferometer; squeezing is then injected; power recycling is added; finally power recycling with squeezing.

port of both a simple Michelson and a power recycled (recycling gain of 10) Michelson interferometer. As shown in Figure 5 the results show that squeezing and power recycling are not only compatible but the effectiveness of the squeezed state is actually enhanced. The major source of loss of the squeezed state was in the optics used to inject the squeezed beam and extract the signal.

This experiment was a vanguard experiment pointing the way for the use of non classical light in interferometers. Whilst it was restricted to shot noise suppression due to the nature of the experiment, future laser interferometers will be limited by quantum noise (shot noise and radiation pressure noise) over most of their frequency band. Noise reduction below the standard quantum limit can be achieved by injecting a squeezed vacuum state in which amplitude and phase noise are correlated. Experiments are now under way using classical modulation signals to demonstrate the principal of quantum noise cancellation (Mow-Lowry 2002). Research on other third generation quantum technologies, such as quantum speed meters and quantum optical springs is also being actively pursued.

\section{High Power Laser Development at UA}

Laser development at the University of Adelaide conducted in support of ACIGA has two main areas of focus: developing high optical power and reducing laser noise. Though the goal of $100 \mathrm{~W}$ of output power seems modest compared to pulsed lasers available today, this power level is unprecedented in a continuous wave laser with the mode quality and frequency stability specified for gravitational wave detector applications. In this section the development of an Nd:YAG (1064 $\mu \mathrm{m})$ laser with virtually diffraction-limited beam quality is described.

Building Power: The basic approach used is to sidepump a slab of the laser medium while controlling temperature gradients in the slab using a combination of forced water heat exchangers and thermoelectric devices. Carefully controlling temperature gradients minimises thermal lensing effects and improves the spatial stability of the output beam.

Temperature gradient control is achieved by cooling the pumped sides of the slab with water while the top and bottom face temperatures are precisely controlled using thermoelectric devices which can either heat or cool the slab as needed.

The slab is pumped by light from laser diodes brought up to the slab via optical fibres (see Figure 6). Each diode laser is wavelength-tuned to the absorption peak of the slab using individual feedback loops. Using this design 


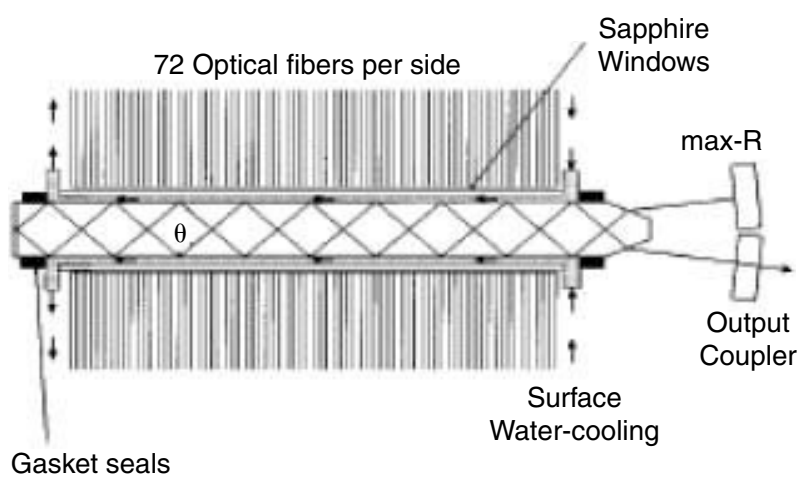

Figure 6 Top view of the side-pumped Nd:YAG slab laser. Master laser light enters through a high-reflectivity mirror (top, right) and undergoes total internal reflection inside the slab, until exiting through an output coupler, right. This light stimulates photon emission inside the slab that is being energised or 'pumped' by diode laser light delivered to the slab by the 72 optical fibres (top and bottom).

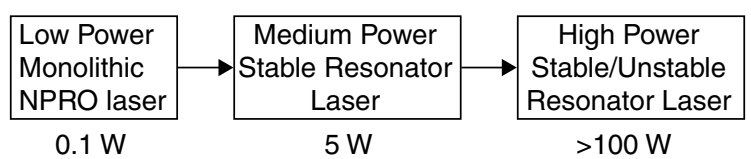

Figure 7 Method of scaling stabilised laser power through injection locking of successive master-slave lasers. The diode-pumped $\mathrm{Nd}$ :YAG laser requires an input light source to stimulate emission. The stability of the first master laser determines the stability of subsequent slave lasers.

and $100 \mathrm{~W}$ of pumping power, a near-diffraction limited $20 \mathrm{~W}$ laser output was produced (Mudge et al. 2000). A scaled-up version is now being tested to produce a target output of $100 \mathrm{~W}$ using $520 \mathrm{~W}$ of pumping power.

Reducing Noise: An ideal interferometer is sensitive only to the phase difference of the two arms and should reject any common-mode fluctuations in the input light. In practice however, laser frequency noise can couple into the output due to unavoidable asymmetry in the interferometer arms. Also, intensity noise can couple into the output through radiation pressure variations between the two arms. Reducing these sources of noise at the source-the input laser-is of prime importance.

Frequency stabilisation is achieved using the PoundDrever-Hall (PDH) technique described by Drever et al. (1983). Due to several practical considerations, PDH stabilisation is only readily applicable to relatively lowpower lasers. Research at UA has therefore centred on injection locking of high-power slave lasers using a stabilised low-power master laser. This method has also been extended to three stages for very high output powers, as illustrated in Figure 7. A $5 \mathrm{~W}$ injection-locked slave laser has been demonstrated (Ottaway et al. 2000) with noise performance suitable for use in gravitational wave detector interferometers. The intention is to use it as a master laser to an even higher power slave laser.

Intensity noise has been investigated as primarily the result of fluctuations in pumping laser intensity. Ottaway et al. (2000) working at UA have also demonstrated the use of intensity stabilisation through feedback on multiemitter high-power diode lasers pumping a high-power slave laser, again with promising results for gravitational wave interferometry.

\section{Test Mass Research at UWA}

Very low loss materials are needed to minimise the internal thermal noise of test masses in gravitational wave detectors. The two leading candidate materials are fused silica and grown sapphire. All currently operating detectors use fused silica as the test mass material. However, intensive research at UWA has focused on sapphire test masses for use in advanced detectors.

Sapphire as a test mass material has several advantages over fused silica. First, sapphire has the lowest acoustic loss of all known materials, having a loss angle $\phi<10^{-8}$ compared to $\phi<10^{-7}$ for fused silica. Secondly, sapphire has a higher sound velocity than fused silica, which results in higher frequency internal modes and thus lower thermal noise. Third, sapphire has a high thermal conductivity which is an advantage in cryogenic detectors (Kuroda et al. 1999; Ju et al. 2002), as well as making it less sensitive to thermal lensing. However, the optical absorption of sapphire is higher than fused silica, typically $20 \mathrm{ppm}$ with some small samples reaching as low as $3 \mathrm{ppm}$. It is believed that proper annealing of the sapphire will reduce the absorption level.

Sapphire is intrinsically birefringent, though this can be overcome by high precision alignment of the test mass position. At UWA, the inhomogeneous birefringence was measured (Benabid et al. 1999), showing that this type of birefringence is actually much smaller than the stressinduced birefringence which would also occur in isotropic materials. Intrinsic birefringence is therefore not seen as a major drawback to sapphire.

Rayleigh scattering in sapphire can also introduce noise in gravitational wave detectors. Measurements showed that the scattering level is about 50 times higher than the fundamental limit predicted by thermodynamic fluctuation theory (Benabid et al. 1998). This is due to the existence of micro point defects in the material. High purity sapphire samples should have lower scattering. It has also been observed that scattering is not uniform over sapphire test masses (Yan et al. 2003). This is a disadvantage to sapphire which must be investigated further.

One important issue for thermal noise is the test mass suspension. Although the gravitational restoring force of a pendulum is lossless, there is still some loss in the material comprising the suspension pivot. The loss angle of the pendulum can be expressed as (Saulson 1990)

$$
\varphi_{\mathrm{p}}=\frac{k_{\mathrm{s}}}{k_{\mathrm{g}}} \varphi=\gamma Q
$$

where $k_{\mathrm{S}}$ is the spring constant of the suspension flexure, $k_{\mathrm{g}}$ is the gravitational spring constant, and $\gamma=k_{\mathrm{s}} / k_{\mathrm{g}}$ is the $\mathrm{Q}$ enhancement factor (also called dilution factor) which 


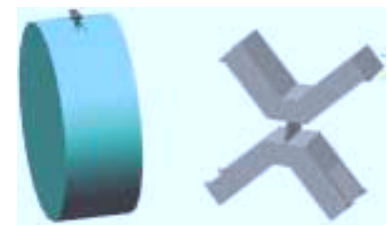

Figure 8 The niobium flexure suspension concept. Internal suspension wire modes are eliminated and the test mass Q-factor optimised by the absence of wires and wire bonding. The metal flexure is robust, and installation is simple. The X-shaped flexure slides into dovetail grooves in the test mass and in the supporting structure above it.

is geometry dependent. $\varphi$ is the loss angle of the suspension material and $Q=1 / \varphi$ is the quality factor. It can be seen that choosing low loss suspension material is essential. Currently, fused silica fibres bonded to a fused silica test mass gives the lowest pendulum loss (Cagnoli et al. 2000). However, bonding fused silica fibre to sapphire has the problem of localised stress due to the mismatch of thermal properties. Besides, fused silica fibre is fragile, prone to moisture damage, and not compatible with cryogenic applications. At UWA, research has centred on an alternative suspension technique - the $\mathrm{Nb}$ flexure module suspension shown in Figure 8.

Niobium was selected as the flexure material because it has the lowest acoustic loss of any metal. It is non-brittle and has a room temperature thermal expansion coefficient compatible with sapphire. It also has excellent yield to Young's modulus ratio (which leads to high $\gamma$ ) and an excellent cryogenic thermal conductivity. The flexure module makes replacement quite simple in case of damage to the suspension. The short membrane flexure has the advantage of avoiding violin string modes which contaminate the gravitational wave observation frequency band. Tests resulted in a pendulum Q-factor of $8 \times 10^{6} \mathrm{using}$ a small monolithic $\mathrm{Nb}$ pendulum, though it is expected to achieve Q-factors of greater than $10^{7}$ when $\gamma$ is optimised (Ju et al. 2001). One issue of flexure module suspension is that it requires a small groove to be cut in the sapphire test mass. This can introduce additional losses into the test mass. However, tests conducted on a small sapphire test mass (Bilenko et al. 2002) show that the degradation is reversible with annealing. A Q-factor of $7 \times 10^{7}$ should be achievable with a $10 \mathrm{~kg}$ test mass.

\section{Data Analysis of the Interferometer Output}

The ACIGA Data Analysis program works in close collaboration with the LIGO Data Analysis program. Since 1999 ACIGA has made significant contributions to the LIGO Data Analysis System (LDAS), in particular, the Data Conditioning API (Blackburn, LIGO document T990002$00-\mathrm{E})$. These range from the infrastructure of the universal data type (UDT) and command syntax implementation, to sophisticated signal processing algorithms such as a system identification theory-based spectral line removal tool. LDAS underpins the search codes used by LIGO to produce astrophysical results from the raw interferometer output.
ACIGA has worked intensively with the LDAS development group for the last three years, meeting weekly by teleconference, exchanging technical emails on a daily basis, and participating in Mock Data Challenges in the US to integrate and scientifically validate software components (Finn, LIGO document T000124-00-E).

ACIGA is a partner in an international exchange of physical environment monitor (PEM) data (Marka, LIGO document G020256-00-D), along with LIGO, GEO, and VIRGO. Environmental variables, such as seismic motion, magnetic field and power grid voltage fluctuations, are sampled continuously and exchanged between the globally separated project sites (see Figure 9(c)). The sampling is GPS synchronised to ensure sub-microsecond precision of event detection. The PEM data sets are searched for cross-correlations, and the ACIGA data analysis program seeks to contribute to the identification and characterisation of terrestrial noise sources that could couple into two or more globally separated gravitational wave detectors. Additionally, the exchange has provided a test-bed for the US-based Network Data Analysis System (NDAS) prototype, which collects and stores environmental data from participating sites for analysis, and allows evaluation of timing and synchronisation issues in advance of an eventual full-scale gravitational wave data exchange. NDAS has recently been upgraded, and is currently being used to move and analyse gravitational wave detector data as part of S2, the 2nd LIGO Science Run, involving the three LIGO detectors, GEO, and TAMA.

The ACIGA data analysis program is based in the Department of Physics at The Australian National University (ANU), with strong ties to the ANU Supercomputer Facility (ANUSF) which houses the multi-terabyte Mass Data Storage System (MDSS) (see Figure 9(c)) and the Australian Partnership for Advanced Computing's National Facility (APAC-NF) (see Figure 9(a)). The MDSS is used to store data from LIGO interferometers and from the local (ANU) environment monitors. In the future it will store data from AIGO.

The APAC-NF is used for general high-performance computing tasks, including Monte Carlo simulations of the performance of networks of gravitational wave detectors. We have used these simulations to determine the optimal configuration for a global network of gravitational wave observatories, in particular, that Western Australia is a uniquely optimal site for an observatory to complete the existing global network (Searle et al. 2002) (see Figure 9(a), bottom).

The ACIGA Data Analysis Cluster (ADAC), which was recently installed in the Department of Physics at ANU (see Figure 9(b)), gives ACIGA a conformant LDAS implementation to analyse LIGO data without duplication of LIGO's investment in the development of data analysis tools. The facility consists of three dual-processor servers managing a Beowulf cluster of eight high-performance nodes, gigabit Ethernet switching, and a terabyte local cache of RAID storage capacity. The cluster has already been used in the characterisation of the performance of 

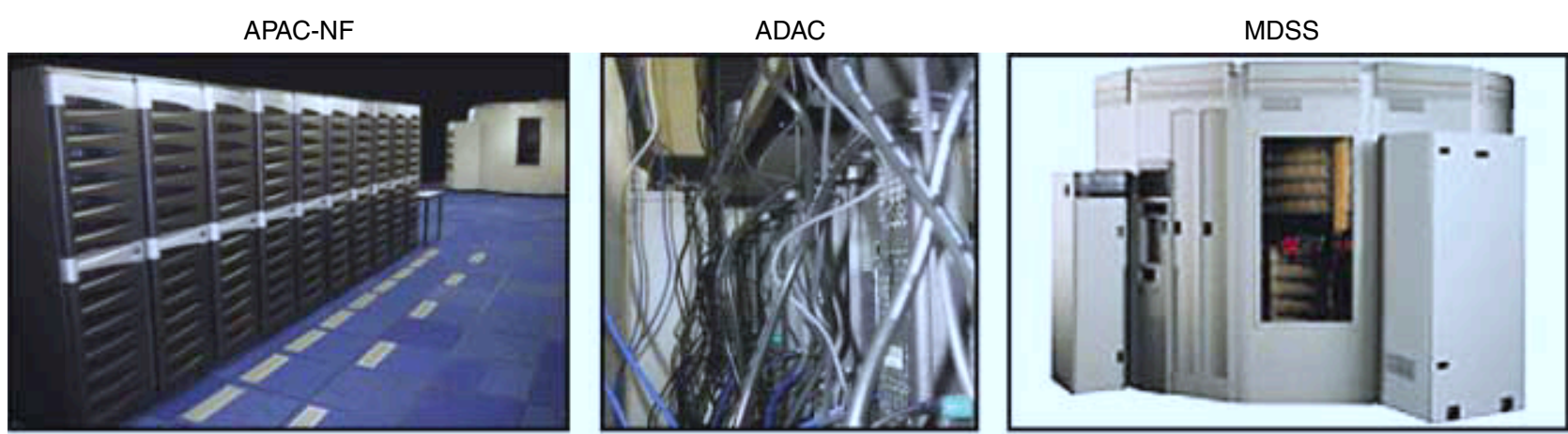

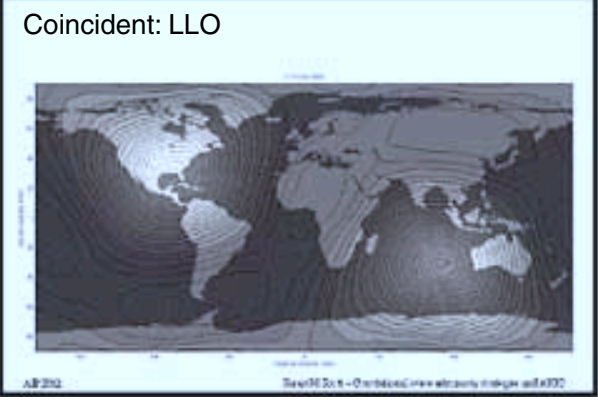

(a)

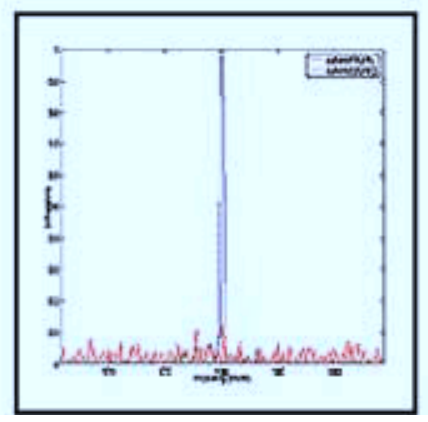

(b)

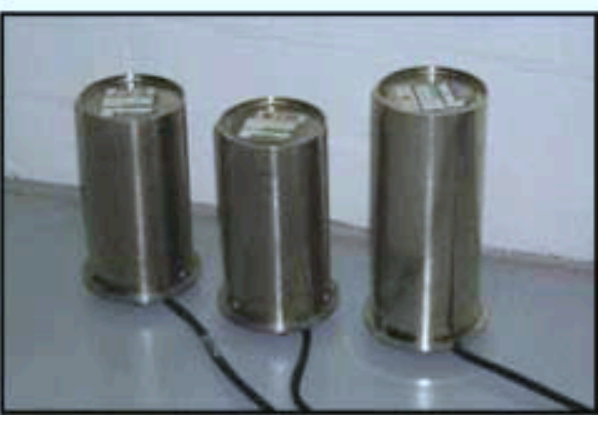

(c)

Figure 9 Top: Computing facilities used by the ACIGA Data Analysis program. (a) The Australian Partnership for Advanced Computing's National Facility (APAC-NF) is used for numerical simulations of global gravitational wave detector networks. (b) The ACIGA Data Analysis Cluster (ADAC) is used to run the LDAS software, and to test new components of the software such as spectral line removal tools. (c) The Mass Data Storage System (MDSS) is used for conversion and storage of large amounts of locally generated environmental data. Bottom: (a) Optimal location for a southern hemisphere gravitational wave observatory intersects Western Australia's Indian Ocean coastal plains. (b) Typical output of a line removal software tool developed at ANU. (c) Seismometers used to collect environmental data used in the PEM international cross-correlation data exchange.

line removal tools on actual LIGO data (Searle 2002) (see Figure 9(b)).

In the terms of the memorandum of understanding between ACIGA and LIGO, ACIGA has access to data taken by the LIGO interferometers during all engineering (i.e. commissioning) and science runs. Access to all of these terabyte-scale datasets is, however, impractical over the Internet. We intend to purchase two LIGO-compatible tape drives for installation at ADAC and Gingin and a multi-terabyte reusable tape cache to facilitate exchange of data between ACIGA and LIGO sites. We also plan the formation of a local archive of LIGO data on the MDSS.

In late 2002 we directly participated in the first science analyses of data taken from LIGO's S1 Science Run. We have worked closely with the LIGO Stochastic Background search group to develop line removal tools. Line removal was integrated into the stochastic background pipeline, and the impact of correlated spectral lines on the stochastic background search codes was assessed (Searle 2002). The analysis culminated in the setting of an upper limit on the strength of a cosmological background of gravitational radiation, the results of which are currently in preparation.

\section{Bringing It All Together: AIGO}

The culmination of the four research areas described above is the AIGO facility near Gingin north of Perth,
Western Australia, Figure 10. Opened in 2000 by the Hon. Richard Court, Premier of Western Australia, the site consists of a $20 \mathrm{~m} \times 20 \mathrm{~m}$ central laboratory, two end-stations, workshops, meeting rooms and accommodation.

As of this writing, leak detection and repair of the test mass vacuum tanks and one $80 \mathrm{~m}$ beam pipe is nearing completion and an enclosure for the lasers and input optics has been built inside the central laboratory. By mid-year 2003, all the research done in ACIGA for the past several years will be brought together for the first fully-suspended high-power optical cavity experiments.

The following section describes the facility in more detail, and some of the research that will be conducted there.

\section{AIGO High Optical Power Test Facility}

The High Optical Power Test Facility (HOPTF) will make use of all the major research achievements of ACIGA in order to provide a platform for a series of experiments slated for mid 2003 through 2005 . The first stage requires a suspended $72 \mathrm{~m}$ cavity in which the substrate of the input test mass (ITM) mirror is inside the optical cavity. An input laser power of about $5 \mathrm{~W}$ will be used. Predictions of thermal lensing effects and substrate absorption will be checked, and tests of cavity locking systems and wavefront distortion control will be made. The second stage requires only that the ITM be reversed so that the reflective side 


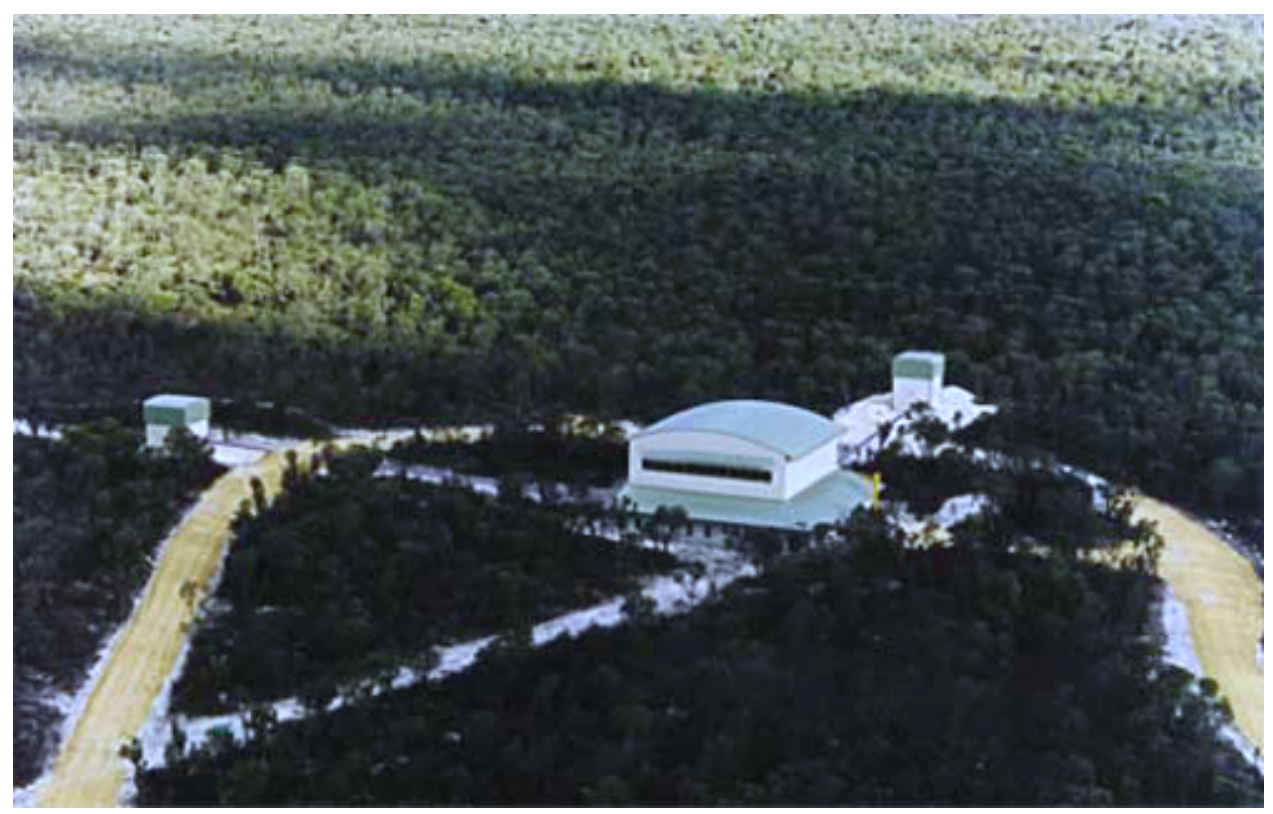

Figure 10 Aerial photo of the AIGO site $80 \mathrm{~km}$ north of Perth. Two $80 \mathrm{~m}$ arms are visible extending from the central laboratory. The site benefits from minimal seismic interference from human activity, and unspoiled natural bushland abounding in biodiversity.
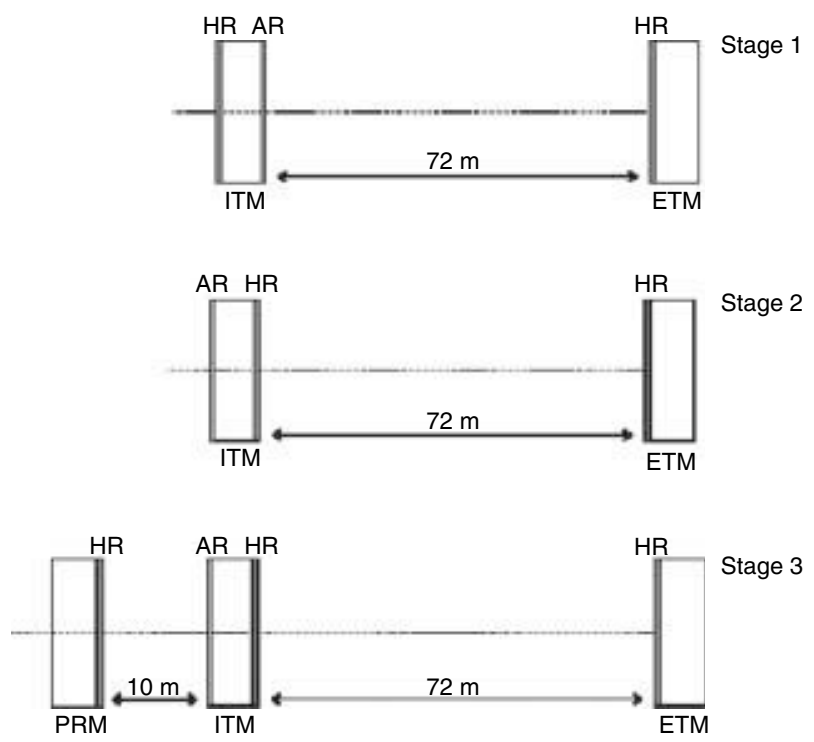

Figure 11 The first three experimental stages for HOPTF. AR and HR designate anti-reflective and high-reflectivity optical coatings, respectively. In Stage 1 the substrate is situated inside the optical cavity in order to investigate thermal lensing. In Stage 2 the ITM optic is reversed. In Stage 3 a power recycling cavity is added, and the input power increased.

faces the cavity. Absorption of the coating will be tested, and the input power will be increased to around 20 to $50 \mathrm{~W}$. The third stage of tests sees the addition of a power recycling mirror (PRM) and a cavity power build-up of around $200 \mathrm{~kW}$. Optical spring effects will be investigated using this configuration (see Figure 11).

This section describes some of the specific research problems to be addressed at AIGO HOPTF as well as some of the specifics of the HOPTF experimental infrastructure. Thermal lensing and radiation pressure/optical spring effect are discussed. Then, details about the data acquisition system, vibration isolation system, and vacuum system are presented.

\section{Thermal Lensing}

Increasing the optical power within the interferometer arm cavities is necessary to reduce shot noise and increase sensitivity, but doing so creates new problems. Thermal lensing and the optical spring effect are two of the problems anticipated for high optical power interferometry.

Thermal lensing is the term given to induced wavefront distortions due to the absorption of significant amounts of power within the transmissive optics of the interferometer (Strain et al. 1994). The absorbed optical power (usually less than $1 \mathrm{~W}$ for the ITM) causes a non-uniform rise in temperature in the optics substrate. The extent of this depends mainly on the energy profile of the absorbed beam and the thermal conductivity of the substrate. The temperature variation (around $1 \mathrm{~K}$ ) changes the optical path length inside the test mass, due in part (about 60\%) to the temperature dependence of the refractive index. Another $35 \%$ comes from simple thermal expansion of the substrate, causing a slight bulge where the beam is incident. An additional $5 \%$ of thermal lensing is attributable to a synergy of these two effects, namely an additional refractive index change as a result of induced mechanical stress, due to the fact that the thermal expansion is non-uniform (the photoelastic effect). For a laser beam of $3 \mathrm{~kW}$ and a transmissive sapphire test mass, the optical path length change measured across the beam diameter is typically around $25 \mathrm{~nm}$. 
One consequence of thermal lensing is mode coupling. Due to non-sphericity of the induced thermal lens the beam spatial fundamental mode is cross-coupled into higher modes which are non-resonant within the cavity, resulting in decreased power in the cavity, added noise at the photodetector, and an offset in control feedback loops. Furthermore, a strong thermal lens can also reduce fringe contrast at the photodetector and make the resonant cavity unstable, because the radius of curvature, and therefore the focal length of the mirrors, has actually changed.

One way to control thermal lensing is to reduce the thermal gradient inside the optics (Lawrence et al. 2002). A heating device may be added close to the material which heats the edge of the optics through radiative heat transfer. Physical contact, although more efficient for heat transfer, is not considered because it degrades the thermal noise performance of the test mass. This method is suitable for weak to moderate thermal lensing.

For strong thermal lensing, a compensation plate may be used. A compensation plate is a fused silica plate (chosen for high transparency and low thermal conductivity) with a heating wire wrapped around it. The plate is placed in the cavity near the optics and compensates for thermal lensing by creating the opposite thermal gradient. The temperature of the wire is adjusted to make the spatial variation in optical path length of the compensating plate exactly opposite to that of the optics.

\section{Radiation Pressure and Optical Spring Effect}

It is well known that light carries momentum as well as energy. Radiation pressure can be interpreted as the transfer of momentum from photons as they interact with a surface. This effect is usually quite small. However, if the incident light power is very high, such as in the planned Advanced LIGO (Gustafson et al., LIGO document T990080-00-D) gravitational wave detector and HOPTF, this effect is substantial.

For instance, in the planned final stage of HOPTF, the laser power circulating in the arm cavity will be $200 \mathrm{~kW}$. At this level of laser power the radiation pressure applied on the mirror is about $1.3 \mathrm{mN}$, sufficient to deflect the UWA pre-isolator by $20 \mu \mathrm{m}$, which is 40 times the free spectral range of the cavity.

Because the mirror motion will modulate the intensity inside an optical cavity, the resultant change in radiation pressure will act back on the mirrors. Thus, the light in the optical cavity acts like an optical spring. The spring constant depends on the frequency offset between the laser and the cavity resonance.

The optical spring phenomenon leads to a correlation between radiation pressure fluctuations and intensity fluctuations. This arises because a radiation pressure fluctuation exerts a force which changes the cavity length, which in turn changes its resonance condition and hence the light intensity inside the cavity. At the quantum level the noise correlations can be used to suppress the total noise below the SQL (Buonanno \& Chen 2001).
At the classical level, the cross-coupling between intensity and radiation pressure can lead to various effects which include amplification or attenuation of perturbations, instabilities, bistability, and mechanical frequency tuning effects. Their effects depend on the parameters of the optical-mechanical system. In experiments to date most observations have focused either on the real or the imaginary component of the optical spring.

Many optical spring phenomena have been observed. Bistability was first observed by Dorsel et al. (1983). Very recently, Tucker et al. (2002) examined the radiation pressure effect in a microscopically scaled tuneable FabryPérot cavity in which the real component of the optical spring contributed substantially to the mechanical resonant frequency. Strong frequency tuning and hysteresis were observed. Cohadon et al. (1999) successfully cooled the Brownian motion of a mirror via the radiation pressure of a laser beam.

In association with the UWA niobium bar gravitational wave detector Tobar (1996) investigated the electromechanical coupling due to the microwave radiation forces across the transducer. He developed a detailed model for the phenomenon which includes both the real and imaginary components of the electromagnetic spring. The real part changes the stiffness of the cavity while the imaginary part changes its quality factor Q. Results showed detailed agreement between theory and experiment. Both frequency tuning (real component) and cold damping (imaginary component) were observed in agreement with theory, and the phenomena were utilised to substantially aid in the operation of the detector (Blair et al. 1995). Chang et al. (1997) examined the microwave radiation pressure induced expansion of a solid dielectric resonator.

We proposed to undertake a similar comprehensive investigation of the phenomena in suspended optical cavities and to determine whether the effects can be utilised to stabilise and cold damp mechanical resonant modes.

The work will lead the way towards the exciting goal of making a gravitational wave detector able to exceed the SQL based on the theory of Buonanno \& Chen (2001).

\section{Data Acquisition, Transfer, and Analysis}

The foundations of a system for data collection and transfer are well established at the HOPTF. A prototype data collection system, using National Instruments hardware and software, is installed and capable of logging 16 analogue channels. This data includes three axes of magnetometer data, three axes of electromagnetic pulse data, two-axis seismic data at two different locations, vertical seismic data from each test mass, wind speed and direction, and power grid noise data. The system will be scaled up in accordance with future requirements.

Measures have been taken to ensure the time accuracy of the data collection system. An ultra-stable rubidium atomic clock is used to regulate and synchronise the data sampling frequency of all channels to a high precision. A GPS receiver is installed on the roof of the main building, 


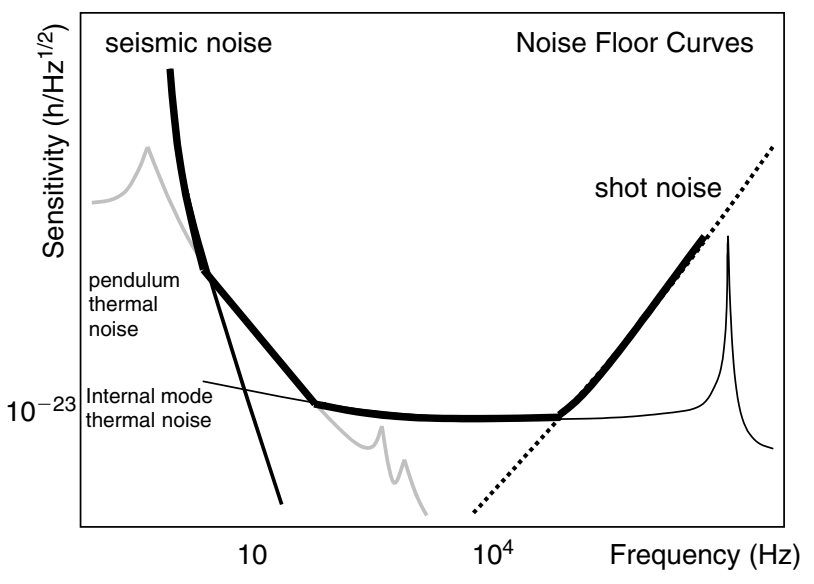

Figure 12 Graph of typical noise sources of an interferometer gravitational wave detector. At low frequencies, seismic noise forms an impenetrable wall defining the detection band. The detection band noise floor is defined by thermal noise of the suspension (pendulum) and of the test mass. The high-frequency detection limit is defined by photon shot noise, essentially statistical counting error.

and will allow data to be time-stamped to an accuracy of less than $400 \mathrm{~ns}$.

The purchase of a server-grade computer is currently planned for the site. The computer will be configured to collect, process and store logged data in an automated fashion, and will be capable of running the LDAS software.

Even with the recent addition of a satellite broadband connection at AIGO $(512 \mathrm{kbps})$, live data transfer is not yet possible between the AIGO site and ANU where the data will be analysed. A direct wireless link to the UWA campus using $2.4 \mathrm{GHz}$ IEEE $802.11 \mathrm{~b}$ compliant hardware is being investigated which could raise the connection rate to $11 \mathrm{Mbps}$.

\section{The Vibration Isolator}

The lower frequency limit for Earth-based interferometric gravitational wave detectors is established in the first instance by seismic noise. Human activity (including transport, machinery, and even foot traffic), as well as natural processes (ocean waves, wind gusts, animal movements, and tectonic activity), all produce ground vibrations that create low frequency noise of the order of ten billion times stronger than gravitational wave signals. For instance at $100 \mathrm{~Hz}$, the seismic RMS amplitude has a value of about $10^{-10} \mathrm{mHz}^{-1 / 2}$, approximately ten orders of magnitude higher than the desired sensitivity. Eliminating seismic noise is therefore fundamental to achieving required detector sensitivities. Ideally, a vibration isolator would substantially eliminate seismic noise in the detection band and minimise residual motion of the suspended test mass. The mechanical vibration isolation system at AIGO is designed to produce a residual motion of the test mass mirror on the order of nanometres.

Figure 12 shows a typical sensitivity curve for an Earthbased interferometer with appropriate seismic isolation. It highlights the dominance of seismic noise in the low end

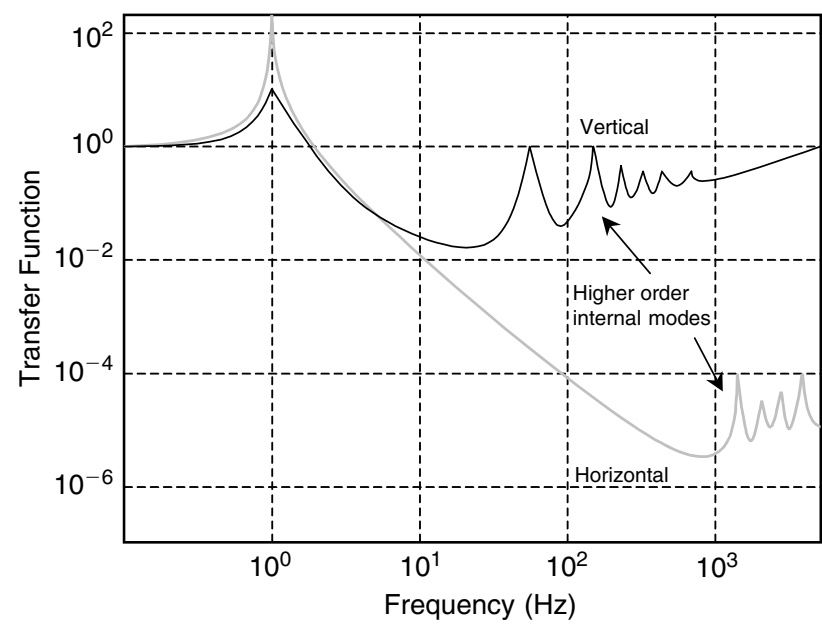

Figure 13 Typical simulated transfer functions of the horizontal and vertical directions of isolation. The vertical scale is the ratio of output to input amplitudes. Note the higher order internal modes; the vertical appears at lower frequency than the horizontal. The Euler type vertical springs have been implemented into the AIGO isolation stack to eliminate these modes from the detection band.

of the detection band, and also indicates that thermal noise in the isolator is the next strongest noise source.

The complete vibration isolation system consists of pendulums, masses, and springs arranged in a chain of several stages that are suspended from a pre-isolation stage. The purpose of an isolation system is to attenuate the transmission of seismic vibrations to the test mass to an acceptably low level. An isolation stack consisting of $n$ stages improves the performance by a factor of $f^{n}$ at frequencies above the last resonance (the 'corner frequency'). The isolator is designed to have its normal mode resonant frequencies all well below the lowest detection frequency while every attempt is made to place the higher internal modes of the isolator above the detection band (see Figure 13).

Pendulums are used as horizontal passive vibration isolators because they are good attenuators of vibration at frequencies above their resonance. They can also have high internal frequency modes. But for isolation in the vertical direction, some kind of spring must be used. Energy from the vibrational motion between the suspension point and the test mass has to somehow be momentarily stored. Suspending massive loads requires the storage of more energy. For a soft spring to store it, the spring must displace a great distance, and therefore must be very large. This reduces the frequencies of the spring's internal modes and places more of them directly in the detection band.

The solution to this problem is to store the vast majority of the gravitational energy in a rigid structure, and the minuscule, residual amount due to small fluctuations in a very soft (low stiffness) spring. This can be accomplished using a thin, flat structure loaded in axial compression which rigidly supports a load up to its buckling limit, then collapses into a low-stiffness buckled spring via Euler buckling. 


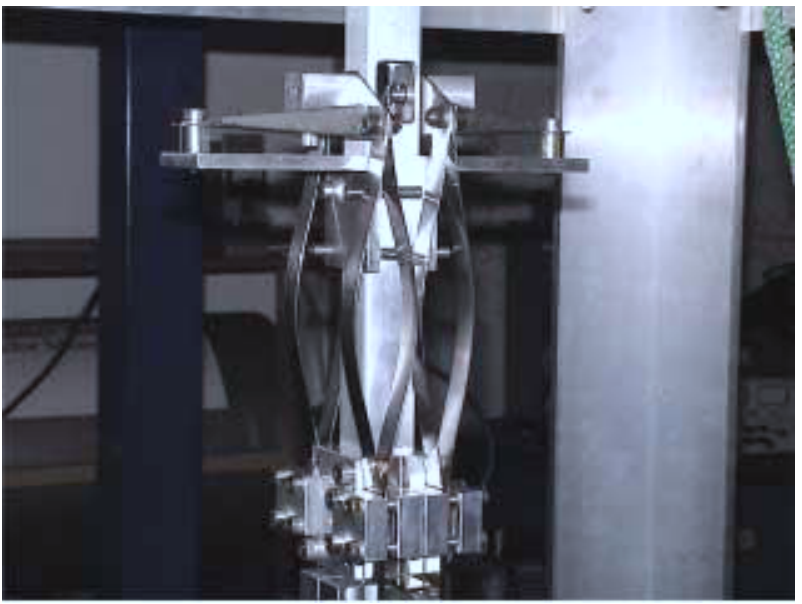

Figure 14 Euler springs shown here can support about $100 \mathrm{~kg}$ with a bounce frequency of less than $1 \mathrm{~Hz}$. They are about $200 \mathrm{~mm}$ long, and have their first internal resonance at about $4 \mathrm{kHz}$. The configuration shown is typical of the ACIGA vertical vibration isolation stages, with springs under compression loaded from the top by a pair of pendulum wires forming the subsequent horizontal isolation stage.

First introduced by the UWA suspension group, Euler springs have been implemented in the AIGO isolation stack. A Euler spring is a column of spring material that has been compressed beyond its Euler buckling limit. The main advantage in using these springs is that they minimise the spring mass required to support the suspended test mass. This results in having far higher internal modes leaving the detection band clear of resonance peaks. The springs also allow for the construction of more compact forms of $3 \mathrm{D}$ vibration isolators due to their very small size (see Figure 14).

The vibration isolation chain developed for AIGO consists of several stages and is illustrated schematically in Figure 15. The ultra low frequency pre-isolation stage suspends the conventional isolation stages to further reduce residual motion. It uses the principle of multiple inverse pendulums supporting a platform, termed the wobbly table, to achieve very low resonant frequencies in the horizontal direction. A LaCoste pendulum is employed to reduce the vertical spring rate in the pre-isolator. The low spring rate is obtained by effectively incorporating an inverse pendulum effect caused by the torque created by the spring that acts against the mass under the force of gravity. The second section of the pre-isolation stage is essentially a 2D pendulum called the Roberts linkage. Each individual stage of the pre-isolator has a resonant frequency of no more than $100 \mathrm{mHz}$. The main vibration isolation chain consists of a cascade of four horizontal and vertical stages where the last is the mirror control mass stage. They are suspended from each other by simple wire pivots consisting of a wire loop wrapped around a pin attached to the central tube. The amplitude of vibration at the resonant frequency of each pendulum is amplified by an amount determined by its Q-factor. It is thus necessary to implement damping mechanisms into the isolation system. A frame independent method, termed

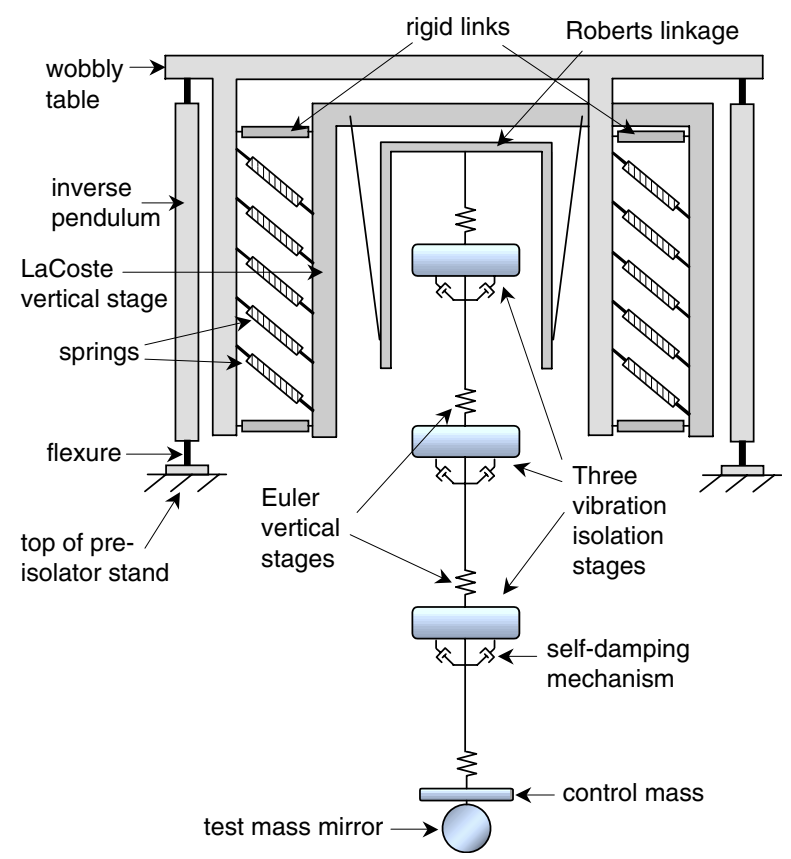

Figure 15 A schematic diagram of the vibration isolation stack for AIGO displaying the three main sections: a pre-isolation stage, multi-stage vibration isolation chain, and the test mass. The preisolator consists of a 2D inverse pendulum horizontal stage, a LaCoste vertical stage, and a Roberts linkage 2D horizontal stage. Suspended from the Roberts linkage stage is the isolation chain consisting of four vertical Euler spring mass stages interspersed with three 2D horizontal self-damping mass-pendulum stages. This extremely compact design utilises less than $3 \mathrm{~m}$ of vertical space and is confined to a $1.8 \mathrm{~m}$ diameter vacuum tank.

self-damping, involves the use of strong rare earth magnets and copper plates spaced closely together inducing eddy currents due to relative motion between them. The coupling of the pendulum with the inertial mass pivoting on the pendulum creates current loops in the copper plates and allows energy to be dissipated.

The final stage of the isolation stack is the test mass control stage. This includes a sapphire test mass mirror suspended from the control mass by a niobium flexure. The complete final stage will be suspended from a vertical Euler stage and will weigh about $40 \mathrm{~kg}$.

\section{Summary}

ACIGA research activities include aspects of the four critical areas for gravitational wave interferometry: advanced configurations and quantum optics; isolation, suspension, and thermal noise; high-power lasers and stabilisation; and data analysis. Closely related to data analysis is the astrophysical research also done in ACIGA studying potential sources of gravitational wave waves and creating expected detection profiles.

ACIGA's activities play a vital role in the international gravitational wave research community. The level of nationwide collaboration, the depth of support, and the range of specialties drawn upon is unprecedented in Australian astrophysics. This effort has already paid dividends in placing Australia in the vanguard of gravitational wave 
research and attracting students and visitors from all over the world. Besides the obvious connection to the future of astronomy, the techniques and new science developed for this project have applications in many other fields, including optical astronomy. The benefits of this program are not limited to just scientific progress. Technology spin-offs and collaborations with Australian industry have already occurred and are expected to continue.

\section{Acknowledgements}

This work is supported by the Australian Research Council, the Department of Education, Science, and Training (DEST), and by LIGO Laboratories.

The authors wish to thank the LIGO ACIGA Advisory Committee: Jordan Camp, Bill Kells, David Ottaway, David Reitze, Benno Willke, and Mike Zucker. Also acknowledged is David Shoemaker for his support of this committee.

The authors also wish to thank the Gravity Waves technical staff at UWA: Ken Field, Peter Hay, Mike Kemp, John Moore, Xiaomei Niu, Tim Slade, Daniel Stone, Vinnie Nguyen, and Xiaolin Wang. Leica Kelly is also thankfully acknowledged for administrative assistance at UWA.

\section{References}

Ando, M., Kawabe, K., \& Tsubono, K. 1997, PhLA, 237, 13

Ando, M., et al. 2001, PhRvL, 86, 3950

Belczynski, K., \& Kalogera, V. 2001, ApJ, 550, L183

Benabid, F., Notcutt, M., Ju, L., \& Blair, D. G. 1998, PhLA, 237, 337

Benabid, F., Notcutt, M., Ju, L., \& Blair, D. G. 1999, OptCo, 167, 7

Bilenko, I. A., Ju, L., Paget, D., \& Blair, D. G. 2002, MeScT, 13, 1173

Billing, H., Maischberger, K., Rüdiger, A., Schilling, R., Schnupp, L., \& Winkler, W. 1979, JPhE, 12, 1043

Blackburn, J. K. The Data Conditioning API's Baseline Requirements, LIGO Document T990002-00-E, http://www.ligo.caltech. edu/docs/T/T990002-00.pdf

Blair, D. G., Ivanov, E. N., Tobar, M. E., Turner, P. J., van Kann, F., \& Heng, I. S. 1995, PhRvL, 74, 1908

Bondu, F., et al. 2002, CQGra, 19, 1829

Buonanno, A., \& Chen, Y. 2001, PhRvD, 64, 042006

Cagnoli, G., Gammaitoni, L., Hough, J., Kovalik, J., McIntosh, S., Punturo, M., \& Rowan, S. 2000, PhRvL, 85, 2442

Caves, C. M. 1980, Phys. Rev. Lett. 45, 75

Chang, S., Mann, A. G., Luiten, A. N., \& Blair, D. G. 1997, PhRvL, 79,2144

Cohadon, P. F., Heidmann, A., \& Pinard, M. 1999, PhRvL, 83, 3174

Coward, D. M., Blair, D. G., Clay, R., \& Mazzitelli, G. 2002a, CQGra, 19, 1871

Coward, D. M., Burman, R. R., \& Blair, D. G. 2002b, MNRAS, 329,411

Cutler, C., \& Thorne, K.S. 2002, preprint gr-qc/0204090

de Vine, G., Shaddock, D. A., \& McClelland, D. E. 2002a, CQGra, 19,1561

de Vine, G., Shaddock, D. A., \& McClelland, D. E. 2002b, OptL, 27, 1507

Djorgovski, S. G., et al. 2001, in Gamma-Ray Bursts in the Afterglow Era: 2nd Workshop, ed. E. Costa, F. Frontera, \& J. Hjorth (Berlin: Springer)

Dorsel, A., McCullen, J. D., Meystre, P., Vignes, E., \& Walther, H. 1983, PhRvL, 51, 1550

Dragicevich, P. M., Blair, D. G., Burman, R. R. 1999, MNRAS, 302,693
Drever, R. W. P. 1982, in Gravitational Radiation, ed. T. Piran \& N. Deruelle (Amsterdam: North Holland), 321

Drever, R. W. P., Hall, T. L., Kowalski, F. V., Hough, J., Ford, G. M., Munley, A. G., \& Ward, H. 1983, ApPhB, 31, 97

Eardley, D. M. 1983, Gravitational Radiation, ed. N. Dereulle \& T. Piran (Amsterdam: North-Holland), 257

Ferrari, V., Matarrese, S., \& Schneider, R. 1999a, MNRAS, 303, 247

Ferrari, V., Matarrese, S., \& Schneider, R. 1999b, MNRAS, 303, 258

Finn, L. S., et al., LDAS Data Conditioning API: Mock Data Challenge \#1, LIGO Document T000124-00-E, http://www.ligo. caltech.edu/docs/T/T000124-00.pdf

Flaminio, R., \& Heitmann, H. 1996, PhLA, 214, 112

Freise, A., et al. 2002, CQGra, 19, 1389

Gray, M. B. 1998, ApOpt, 37, 5886

Gray, M. B., Stevenson, A. J., Harb, C. C., Bachor, H.-A., \& McClelland, D. E. 1996, ApOpt, 35, 1623

Gustafson, E., Shoemaker, D., Strain, K. A., \& Weiss, R. LSC White paper on detector research and development, LIGO document No T990080-00-D, www.ligo.caltech.edu/docs/T/T990080-00.pdf

Hogg, D. W., \& Fruchter, A. S. 1999, ApJ, 520, 54

Ju, L., Baker, M., \& Blair, D. G. 2001, PhLA, 280, 182

Ju, L., Blair, D. G., Bilenko, I., \& Paget, D. 2002, CQGra, 19, 1703

Kalogera, V., Narayan, R., Spergel, D., \& Taylor, J. H. 2001, ApJ, 556,340

Kuroda, K., et al. 1999, IJMPD, 8, 557

Lam, P. K., Ralph, T. C., Buchler, B. C., McClelland, D. E., Bachor, H.-A., \& Gao, J. 1999, JOptB, 1, 469

Landry, M. 2002, CQGra, 19, 1825

Lawrence, R., Zucker, M., Fritschel, P., Marfuta, P., \& Shoemaker, D. 2002, CQGra, 19, 1803

Linthorne, N. P., Veitch, P. J., Blair, D. G., Turner, P. J., Tobar, M. E., \& Mann, A. G. 1990, PhyB, 165, 9

Man, C. N., Shoemaker, D., Manh Pham Tu, \& Dewey, D. 1990, PhLA, 148, 8

Márka, S., et al., Status of Network Data Analysis Server Prototype and Environmental Data Exchange, LIGO Document G020256-00-D, http://www.ligo.caltech.edu/docs/G/G02025600/G020256-00.pdf

McKenzie, K., Shaddock, D. A., McClelland, D. E., Buchler, B. C., \& Lam, P. K. 2002, PhRvL, 88, 231102

Meers, B. J. 1988, PhRvD, 38, 2317

Mizuno, J., Strain, K. A., Nelson, P. G., Chen, J. M., Schilling, R., Rüdiger, A., Winkler, W., \& Danzmann, K. 1993, PhLA, 175, 273

Mow-Lowry, C. 2002, Honours Thesis, The Australian National University

Mudge, D., Ostermeyer, M. O., Veitch, P. J., Munch, J., Middlemiss, B., Ottaway, D. J., \& Hamilton, M. W. 2000, IEEE Journal of Selected Topics in Quantum Electronics, 6, 643

Ottaway, D. J., Veitch, P. J., Hollitt, C., Mudge, D., Hamilton, M. W., \& Munch, J. 2000, ApPhB, 71, 163

Regehr, M. W., Raab, F. J., \& Whitcomb, S. E. 1995, OptL, 20, 1507

Saijo, M., Shibata, M., Baumgarte, T. W., \& Shapiro, S. L. 2001, ApJ, 548, 919

Saulson, P. R. 1990, PhRvD, 42, 2437

Searle, A. C. 2002, Line removal techniques for LIGO data, at GWDAW2002 in Kyoto, Japan, December 2002, http://vega.ess. sci.osaka-u.ac.jp/gwdaw/files/0704.pdf

Searle, A. C., Scott, S. M., \& McClelland, D. E. 2002, CQGra, 19,1465

Shaddock, D. A., et al. 1998, ApOpt, 37, 7995

Shibata, M., Baumgarte, T. W., \& Shapiro, S. L. 2000, ApJ, 542, 453

Shoemaker, D., Fritschel, P., Giaime, J., Christensen, N., \& Weiss, R. 1991, ApOpt, 30, 3133

Stevenson, A. J., Gray, M. B., Bachor, H. A., \& McClelland, D. E. 1993, ApOpt, 32, 3481

Stevenson, A. J., Gray, M. B., Harb, C. C., Bachor, H. A., \& McClelland, D. E. 1995, AuJPh, 48, 971

Strain, K. A., \& Meers, B. J. 1991, PhRvL, 66, 1391 
Strain, K. A., Danzmann, K., Mizuno, J., Nelson, P. G., Rüdiger, A., Schilling, R., \& Winkler, W. 1994, PhLA, 194, 124

Takahashi, R., Mizuno, J., Miyoki, S., \& Kawashima, N. 1994, PhLA, 187, 157

Tobar, M. E. 1996, PhD Thesis, The University of Western Australia Tobar, M. E., Locke, C. R., Heng, I. S., Ivanov, E. N., \& Blair, D. G. 1999, 3rd Amaldi Conference, CP524, 283

Tucker, R. S., Baney, D. M., Sorin, W. V., \& Flory, C. A. 2002, IEEE Journal of Selected Topics in Quantum Electronics, 8, 88 van Putten, M. H. P. M. 2001, PhR, 345,

Weber, J. 1960, PhRv, 117, 306

Weisberg, J. M., \& Taylor, J. H. 1984, PhRvL, 52, 1348

Winterflood, J., Barber, T., \& Blair, D. G. 2002, CQGra, 19, 1639

Yan, Z., Ju, L., Zhao, C., \& Blair, D. G. 2003, Rayleigh scattering study of sapphire cylinder test masses, preprint, upcoming Amaldi 5 conference 